\title{
Cooperative Filters and Control for Cooperative Exploration
}

\author{
Fumin Zhang and Naomi Ehrich Leonard
}

\begin{abstract}
Autonomous mobile sensor networks are employed to measure large-scale environmental fields. Yet an optimal strategy for mission design addressing both the cooperative motion control and the cooperative sensing is still an open problem. We develop strategies for multiple sensor platforms to explore a noisy scalar field in the plane. Our method consists of three parts. First, we design provably convergent cooperative Kalman filters that apply to general cooperative exploration missions. Second, a novel method is established to determine the shape of the platform formation to minimize error in the estimates and a cooperative formation control law is designed to asymptotically achieve the optimal formation shape. Third, we use the cooperative filter estimates in a provably convergent motion control law that drives the center of the platform formation to move along level curves of the field. This control law can be replaced by control laws enabling other cooperative exploration motion, such as gradient climbing, without changing the cooperative filters and the cooperative formation control laws. Performance is demonstrated on simulated underwater platforms in simulated ocean fields.
\end{abstract}

\section{INTRODUCTION}

Missions that require measuring and exploring a scalar field such as a temperature or a salinity field are encountered, for example, in ocean science and meteorology. Since the scalar field is

F. Zhang (corresponding author) is with School of Electrical and Computer Engineering, Georgia Institute of Technology, Savannah, GA 31407. Email: fumin@ece.gatech.edu.

N. E. Leonard is with Department of Mechanical and Aerospace Engineering, Princeton University, Princeton, NJ 08544. Email:naomi@princeton.edu.

We want to thank the MIT and Harvard HOPS group (Pierre Lermusiaux, Allan Robinson, Pat Hayley, Wayne Leslie, in particular) for ocean modeling support. We thank Edward Fiorelli, Francois Lekien, and Pradeep Bhatta for collaborations, suggestions, and discussions. This work was supported in part by ONR grants N00014-02-1-0826, N00014-02-1-0861 N0001404-1-0534, N00014-08-1-1007 and NSF ECCS-0841195 
often distributed across a large area, it takes too many sensors to obtain a snapshot of the field if the sensors are installed at fixed locations. Mobile sensor networks are ideal candidates for such missions: a small number of moving sensor platforms can patrol a large area, taking measurements along their motion trajectories. Exploration activities of great interest include climbing gradients of a scalar field [1], monitoring environmental boundaries [2]-[5], patroling the perimeter of a region or a contour [6]-[12], and providing sampling coverage over a large area [13]-[15]. Various methods are developed and demonstrated in the above references.

Mission design for a mobile sensor network requires a combination of cooperative control and cooperative sensing. This is because the nature and quality of collected information are coupled with the motion of the sensor platforms. Therefore, the challenges in developing successful sensing algorithms are complementary to those addressed in earlier work on distributed but fixed wireless sensor networks (c.f. review articles [16], [17]). Recent theoretical and experimental developments suggest that a balance between data collection and feasible motion is key to mission success [15], [18], [19]. Finding an optimal strategy is a challenging task.

In this paper, we present a general Kalman filter design for mobile sensor networks to perform cooperative exploration missions. Exploration missions are frequently encountered in environmental applications where the mobile sensor platforms are commanded to measure an unknown scalar field corrupted by (correlated) noise. Since each platform can only take one measurement at a time, the platforms should move in a formation or a cluster to estimate local structures of the field.

The Kalman filter combines sensor readings from formation members to provide estimates for the field value and the gradient. A separate cooperative filter is developed to estimate the Hessian. We demonstrate that the formation shape can be made adaptive to minimize the error covariance of the estimate produced by the cooperative Kalman filter. We prove a set of sufficient conditions that the formation and its motion need to satisfy to achieve the convergence of the Kalman filter. Derivation of these sufficient conditions is based on fundamental results connecting controllability and observability of a (time-varying) filtering system to its convergence in [20][22]. More recent developments in [23]-[25] have relaxed the conditions for convergence of Kalman filters to stabilizability and detectability, with even weaker conditions for some special cases. In this paper, we develop the sufficient conditions based on controllability and observability conditions because the resulting constraints on formation design are already mild enough, hence 
are acceptable in typical applications.

Kalman filtering for mobile sensor network applications has received recent attention in the literature. In [26], a distributed Kalman filter method was proposed to decompose a high-order central Kalman filter into "micro" filters computable by each sensor node. The estimates made by each node are then combined using consensus filters [27]. A similar approach is taken in [28] to address target tracking and coverage problems. Another type of Kalman filter design is proposed in [29] where the entire field is partitioned into cells and the movement of agents is controlled to maximize collected information. The above contributions assume that a (dynamic) model for a planar field is known to all nodes, hence each individual is able to compute a Kalman filter. Accordingly, the goal there is to implement a distributed algorithm on many sensor nodes to improve tracking or mapping precision.

For the cooperative exploration problem, on the other hand, the field is completely unknown; a Kalman filter can only be computed by combining readings across platforms. The interest here is to take advantage of the Kalman filter design in order to use a minimum number of sensor platforms to navigate in the unknown scalar field and reveal its structure, e.g., to follow level curves or gradients. In [30], an adaptive scheme using a Kalman filter is developed for interpolating data to construct a scalar field. This contribution addresses different problems than in this paper and is complementary to our results.

In concert with our cooperative filter development, we design provable cooperative control laws to stabilize desired formation shape and motion. There exist many contributions on cooperative formation control that are closely related to graph theory, c.f. [31]-[35], to name only a few. In this paper, we employ a different approach based on geometric reductions. The Jacobi transform [36]-[38] is applied to decouple the motion of the formation center from the motion of the formation shape. Hence the control effort can also be decoupled into control for the formation shape and control for the formation center. We design the shape control and the center control separately and then combine them to get the overall control. The key benefit of this approach is that it allows us to design formation shape control to improve performance of the Kalman filter. The center of the formation can be controlled to perform gradient climbing, level curve tracking, or other motions while the Kalman filter and formation control remains the same. For this reason we name the Kalman filter associated with the formation control in this paper the cooperative Kalman filter. 
In this paper we control the center of the formation to track level curves of a scalar field corrupted by noise; this is a collaborative exploration behavior that reveals structure in the unknown field. The tracking control method is developed to steer the center to follow its projection on a curve. This strategy was first reported in [39], [40] and has been applied to curve tracking for mobile robots, c.f. some recent developments in [41], [42]. A differential geometric approach was developed in [6] which extended the tracking method to three-dimensional curves. The tracking control law in the present paper is a generalization of the differential geometric results to the case of two-dimensional level curve tracking. The control law allows a formation to smoothly find and follow any desired regular level curve with proved convergence.

The organization of this paper is as follows. In Section II, we derive the information dynamics of a typical platform formation that moves in a planar scalar field. In Section III, Kalman filtering techniques are applied to the information dynamics. We establish sufficient conditions for the cooperative Kalman filter to converge. We also show that the formation shape can be made adaptive to minimize the error covariance of the estimates produced by the cooperative Kalman filter. In Section IV, we provide a method to estimate the Hessian that is necessary for the cooperative Kalman filter. Formation shape and orientation control laws are derived based on the Jacobi transform in Section V. In Section VI, a steering control law is designed to control the center of the formation to follow level curves of a planar scalar field. We demonstrate the cooperative Kalman filter and the cooperative control law in a simulated ocean temperature field in Section VII. A summary and discussion for future directions are presented in Section VIII.

\section{INFORMATION DYNAMICS OF COOPERATIVE EXPLORATION}

In this section, we define the cooperative exploration problem and introduce the corresponding information dynamic model. Let $z(\mathbf{r})$ where $\mathbf{r} \in \mathbb{R}^{2}$ be a smooth scalar field in the plane that is unknown. In most practical situations, since the field is corrupted by noise and the sensing devices are imperfect, it is difficult to estimate the field value using a single sensor platform. The key idea for mobile sensor networks is to employ multiple moving sensor platforms to obtain the necessary estimates cooperatively and reduce noise. This requires the platforms to be in a formation, moving and collecting information simultaneously.

In most applications, the sensor measurements are taken discretely over time. This is because the spatial range of the scalar field is usually very large. Hence very small scale fluctuations in 
the field should be filtered out as noise. Let the moment when new measurements are available be $t_{k}$ where $k$ is an integer index. To simplify the derivation, we do not consider the asynchronicity in the measurements; we assume that all platforms have new measurements at time $t_{k}$. In reality, when there exists asynchronicity, the technique we develop can still be applied with slight modifications.

Let the positions of the sensor platforms at time $t_{k}$ be $\mathbf{r}_{i, k} \in \mathbb{R}^{2}$ where $i=1,2, \ldots, N$. We assume that the measurement taken by the $i$ th platform is modeled as

$$
p_{i, k}=z\left(\mathbf{r}_{i, k}\right)+w\left(\mathbf{r}_{i, k}\right)+n_{i, k}
$$

where $z\left(\mathbf{r}_{i, k}\right)$ is the value of the field at $\mathbf{r}_{i, k}, n_{i, k} \sim \mathcal{N}\left(0, \sigma_{i}^{2}\right)$ are i.i.d. Gaussian noise, and $w\left(\mathbf{r}_{i, k}\right)$ are spatially correlated Gaussian noise. We define the following $N \times 1$ vectors:

$$
\mathbf{p}_{k}=\left[p_{i, k}\right], \mathbf{z}_{k}=\left[z\left(\mathbf{r}_{i, k}\right)\right], \mathbf{n}_{k}=\left[n_{i, k}\right], \mathbf{w}_{k}=\left[w\left(\mathbf{r}_{i, k}\right)\right]
$$

and assume that $\mathbf{n}_{k}$ and $\mathbf{w}_{k}$ are stationary, i.e., their statistics are time invariant.

Remark 2.1: By convention in ocean and atmospheric sciences, modeling a physical field as a smooth field $z(\mathbf{r})$ plus a spatially correlated random field $\mathbf{w}(\mathbf{r})$ is often desired to separate larger and smaller scale phenomena. The assumptions we impose here are idealizations for physical scalar fields. In addition, the smoothness of field $z(\mathbf{r})$ helps in developing Hessian filters and motion control laws in later sections.

We define the problem of cooperative exploration, as a special class of mapping problems, as follows:

Problem 2.2: Given the statistics of the noise $\mathbf{n}_{k}$ and $\mathbf{w}_{k}$, co-design cooperative motion and filtering that utilize collected measurements $\mathbf{p}_{k}$ for mobile sensor platforms so that an estimate for the field $z(\mathbf{r})$ that minimizes an error metric $J$ can be obtained.

The choice of the error metric $J$ depends on application. In this paper, $J$ is chosen to be the mean square error over spatial domain.

To solve this problem, there is no need to take measurements at every point in the plane. Sufficient knowledge of the field can be gained by measuring the field value $z$, the gradient $\nabla z$, and Hessian $\nabla^{2} z$ at locations well distributed across the plane and then interpolating the field. Note that this problem can be defined for a time-varying field i.e. $z(\mathbf{r}, t)$. In this paper, we address the time-invariant case. 
We address Problem 2.2 by deriving rigorous tools that are particularly useful when there exist smaller regions within the global area with unknown features that require high resolution sampling; we do not attempt to address Problem 2.2 globally in this paper. Our approach includes one development that focuses on minimizing the local error collectively using the cooperative Kalman filter and coordinated motion that controls the shape of the formation. The other development focuses on a cooperative exploration behavior, namely formation motion control for level curve tracking. This contributes to reducing error at a somewhat larger scale than the filtering and even can contribute to global reduction in error if multiple formations are distributed throughout the region. Central to our approach is the decoupling of the two developments, i.e., formation motion control can be designed independently from formation shape control and the cooperative Kalman filtering. Indeed the level curve tracking can be replaced or augmented with one or more other collaborative exploration behaviors, such as wide-area coverage and gradient climbing, to aid in global error minimization; further, because of the decoupling these can be implemented without affecting the local error minimization.

The function $z\left(\mathbf{r}_{i, k}\right)$ can be locally approximated by a Taylor series. Let $\mathbf{r}_{\mathrm{c}, k}$ be the center of the platform formation at time $t_{k}$, i.e., $\mathbf{r}_{\mathrm{c}, k}=\frac{1}{N} \sum_{i=1}^{N} \mathbf{r}_{i, k}$. If $\mathbf{r}_{i, k}$ is close to $\mathbf{r}_{\mathrm{c}, k}$, then it is sufficient to use the Taylor series up to second order. Let $z_{i, k}=z\left(\mathbf{r}_{i, k}\right)$, then

$$
z_{i, k} \approx z\left(\mathbf{r}_{\mathrm{c}, k}\right)+\left(\mathbf{r}_{i, k}-\mathbf{r}_{\mathrm{c}, k}\right)^{T} \nabla z\left(\mathbf{r}_{\mathrm{c}, k}\right)+\frac{1}{2}\left(\mathbf{r}_{i, k}-\mathbf{r}_{\mathrm{c}, k}^{T}\right) \nabla^{2} z\left(\mathbf{r}_{\mathrm{c}, k}\right)\left(\mathbf{r}_{i, k}-\mathbf{r}_{\mathrm{c}, k}\right)
$$

for $i=1,2, \ldots, N$. We are interested in estimates of $z\left(\mathbf{r}_{\mathrm{c}, k}\right), \nabla z\left(\mathbf{r}_{\mathrm{c}, k}\right)$, and $\nabla^{2} z\left(\mathbf{r}_{\mathrm{c}, k}\right)$. In addition to providing insights on the structure of the scalar field, these estimates are also used in the steering control for the center of the formation, as shown later in Section VI.

\section{A. The Measurement Equations}

Let $\mathbf{s}_{k}=\left[z\left(\mathbf{r}_{\mathrm{c}, k}\right), \nabla z\left(\mathbf{r}_{\mathrm{c}, k}\right)^{T}\right]^{T}$. Let $C_{k}$ be the $N \times 3$ matrix defined by

$$
C_{k}=\left[\begin{array}{cc}
1 & \left(\mathbf{r}_{1, k}-\mathbf{r}_{\mathrm{c}, k}\right)^{T} \\
\vdots & \vdots \\
1 & \left(\mathbf{r}_{N, k}-\mathbf{r}_{\mathrm{c}, k}\right)^{T}
\end{array}\right]
$$

Let $D_{k}$ be the $N \times 4$ matrix with its $i$ th row vector defined by $\frac{1}{2}\left(\left(\mathbf{r}_{i, k}-\mathbf{r}_{\mathrm{c}, k}\right) \otimes\left(\mathbf{r}_{i, k}-\mathbf{r}_{\mathrm{c}, k}\right)\right)^{T}$ where $\otimes$ is the Kronecker product. For any $2 \times 2$ matrix $H$, we use the notation $\vec{H}$ to represent 
a column vector defined by rearranging the elements of $H$ as follows

$$
\vec{H}=\left[H_{11}, H_{21}, H_{12}, H_{22}\right]^{T} .
$$

Then the Taylor expansions (3) for all sensor platforms near $\mathbf{r}_{\mathrm{c}, k}$ can be re-written in a vector form as

$$
\mathbf{z}_{k}=C_{k} \mathbf{s}_{k}+D_{k} \vec{\nabla}^{2} z\left(\mathbf{r}_{\mathrm{c}, k}\right)
$$

where $\vec{\nabla}^{2} z\left(\mathbf{r}_{\mathrm{c}, k}\right)$ is a $4 \times 1$ column vector obtained by rearranging elements of the Hessian $\nabla^{2} z\left(\mathbf{r}_{\mathrm{c}}, k\right)$ as defined by (5).

Suppose that $\vec{H}_{\mathrm{c}, k}$ is an estimate for the Hessian $\vec{\nabla}^{2} z\left(\mathbf{r}_{\mathrm{c}, k}\right)$ in vector form. Equation (1) can now be written as

$$
\mathbf{p}_{k}=C_{k} \mathbf{s}_{k}+D_{k} \vec{H}_{\mathrm{c}, k}+\mathbf{w}_{k}+D_{k} \mathbf{e}_{k}+\mathbf{n}_{k}
$$

where $\mathbf{e}_{k}$ represents the error in the estimate of the Hessian. Let $W_{k}=E\left[\mathbf{w}_{k} \mathbf{w}_{k}^{T}\right], U_{k}=E\left[\mathbf{e}_{k} \mathbf{e}_{k}^{T}\right]$, and $R_{k}=E\left[\mathbf{n}_{k} \mathbf{n}_{k}^{T}\right]$. The noise $\mathbf{w}_{k}$ is "colored" because it originates from the spatial correlation of $\mathbf{w}(\mathbf{r})$. Let $E\left[\mathbf{w}_{k} \mathbf{w}_{k-1}^{T}\right]=V_{k}$. We suppose that $W_{k}, R_{k}$ and $V_{k}$ are known once the positions of the platforms are known. This assumption is reasonable in ocean and meteorology applications since the statistical properties of ocean fields and atmospheric fields are usually known from accumulated observational data over a long period of time. We also assume that $U_{k}$ determined by the accuracy of the Hessian estimation algorithm is known.

\section{B. The State Dynamics}

As the center of the formation moves, the states $\mathbf{s}_{k}=\left[z\left(\mathbf{r}_{c, k}\right), \nabla z\left(\mathbf{r}_{c, k}\right)^{T}\right]^{T}$ evolve according to the following equations:

$$
\begin{aligned}
z\left(\mathbf{r}_{c, k}\right) & =z\left(\mathbf{r}_{c, k-1}\right)+\left(\mathbf{r}_{c, k}-\mathbf{r}_{c, k-1}\right)^{T} \nabla z\left(\mathbf{r}_{c, k-1}\right) \\
\nabla z\left(\mathbf{r}_{c, k}\right) & =\nabla z\left(\mathbf{r}_{c, k-1}\right)+H_{c, k-1}\left(\mathbf{r}_{c, k}-\mathbf{r}_{c, k-1}\right) .
\end{aligned}
$$

Let $\mathbf{h}_{k-1}=\left[0, E\left[H_{c, k-1}\left(\mathbf{r}_{c, k}-\mathbf{r}_{c, k-1}\right)\right]^{T}\right]^{T}$ and $A_{k-1}^{\mathrm{s}}=\left[\begin{array}{cc}1 & \left(\mathbf{r}_{c, k}-\mathbf{r}_{c, k-1}\right)^{T} \\ 0 & I_{2 \times 2}\end{array}\right]$. We then rewrite (8) as

$$
\mathbf{s}_{k}=A_{k-1}^{\mathrm{s}} \mathbf{s}_{k-1}+\mathbf{h}_{k-1}+\boldsymbol{\epsilon}_{k-1}
$$

where we have introduced the $N \times 1$ noise vector $\epsilon_{k-1}$ which accounts for positioning errors, estimation errors for the Hessians, and errors caused by higher order terms omitted from the 
Taylor expansion. We assume that $\epsilon_{k-1}$ are i.i.d Gaussian with zero mean and known covariance matrix $M_{k-1}$ that is positive definite.

Remark 2.3: We note that the assumption that $\epsilon_{k-1}$ is i.i.d Gaussian with zero mean may be unrealistic. Simulation or physical data will help to validate the assumption.

\section{The Noise Dynamics}

The noise $\mathbf{w}_{k}$ in the measurement equation (7) is colored. The standard technique (c.f. [43]) to handle this issue is to model $\mathbf{w}_{k}$ as

$$
\mathbf{w}_{k}=A_{k-1}^{\mathrm{w}} \mathbf{w}_{k-1}+\boldsymbol{\eta}_{k-1}
$$

where $\boldsymbol{\eta}_{k-1}$ is white noise with positive definite correlation matrix $Q_{k}=E\left[\boldsymbol{\eta}_{k} \boldsymbol{\eta}_{k}^{T}\right]$. Because

$$
\begin{aligned}
V_{k} & =E\left[\mathbf{w}_{k} \mathbf{w}_{k-1}^{T}\right]=A_{k-1}^{\mathrm{w}} E\left[\mathbf{w}_{k-1} \mathbf{w}_{k-1}^{T}\right]=A_{k-1}^{\mathrm{w}} W_{k-1} \\
W_{k} & =E\left[\mathbf{w}_{k} \mathbf{w}_{k}^{T}\right]=A_{k-1}^{\mathrm{w}} W_{k-1}\left(A_{k-1}^{\mathrm{w}}\right)^{T}+Q_{k-1},
\end{aligned}
$$

we have

$$
\begin{aligned}
A_{k-1}^{\mathrm{w}} & =V_{k} W_{k-1}^{-1} \\
Q_{k-1} & =W_{k}-A_{k-1}^{\mathrm{w}} W_{k-1}\left(A_{k-1}^{\mathrm{w}}\right)^{T}
\end{aligned}
$$

Remark 2.4: State equation (9) reveals the major difference between the cooperative exploration problem considered in this paper and the tracking/coverage problems considered in [26], [28], [29]. Equation (9), fundamental to the cooperative exploration problem, is only valid for the formation and does not make sense for each individual node, since $A_{k-1}^{\mathrm{s}}$ and $\mathbf{h}_{k-1}$ depend on the location of all platforms in the formation. Therefore, the distributed Kalman filter algorithms for tracking and coverage in [26], [28], and [29], which achieve consensus between nodes and increase computation efficiency, are not applicable here. The central problem here is to use the minimum number of platforms with coordinated motion to estimate the field. For this purpose, we design the cooperative Kalman filter in the next section.

\section{The CoOperative Kalman Filter}

We observe from the information dynamics modeled by (9), (10), and (7) that if the Hessian related term $\mathbf{h}_{k-1}$ is known for all $k$, then the system belongs to the category for which Kalman 
filters can be constructed. In Section IV we show that $\mathbf{h}_{k-1}$ can be estimated. Thus standard procedures can be followed to obtain a Kalman filter, which will be called the cooperative Kalman filter because it can only be computed by a formation and its performance depends on the configuration of the formation. Our main contribution in this section is to establish sufficient conditions that a formation must satisfy for the cooperative Kalman filter to converge.

\section{A. Cooperative Kalman Filter Equations}

The equations for Kalman filters are obtained by canonical procedures, the formulas are derived following textbooks [43]-[45]. For the sake of clarity and convenience for later references we list those formulas for the case when $\mathbf{w}_{k}=0$ as below:

(1) the one-step prediction,

$$
\mathbf{s}_{k(-)}=A_{k-1}^{\mathrm{s}} \mathbf{s}_{k-1(+)}+\mathbf{h}_{k-1}
$$

(2) error covariance for the one-step prediction,

$$
P_{k(-)}=A_{k-1}^{\mathrm{s}} P_{k-1(+)} A_{k-1}^{s T}+M_{k-1}
$$

(3) optimal gain,

$$
K_{k}=P_{k(-)} C_{k}^{T}\left[C_{k} P_{k(-)} C_{k}^{T}+D_{k} U_{k} D_{k}^{T}+R_{k}\right]^{-1}
$$

(4) updated estimate,

$$
\mathbf{s}_{k(+)}=\mathbf{s}_{k(-)}+K_{k}\left(\mathbf{p}_{k}-C_{k} \mathbf{s}_{k(-)}-D_{k} \vec{H}_{\mathrm{c}, k}\right) ;
$$

(5) error covariance for the updated estimate,

$$
P_{k(+)}^{-1}=P_{k(-)}^{-1}+C_{k}^{T}\left[D_{k} U_{k} D_{k}^{T}+R_{k}\right]^{-1} C_{k} .
$$

Here we use subscript $(-)$ to indicate predictions and $(+)$ to indicate updated estimates.

In order to design a Kalman filter with colored measurement noise $\mathbf{w}_{k}$, a well-known method devised in [46] can be applied by defining a new measurement $\widetilde{\mathbf{p}}_{k}$ as $\widetilde{\mathbf{p}}_{k}=\mathbf{p}_{k}-A_{k-1}^{w} \mathbf{p}_{k-1}$. This gives a new equation for measurements:

$$
\begin{aligned}
\widetilde{\mathbf{p}}_{k} & =\left(C_{k} A_{k-1}^{\mathrm{s}}-A_{k-1}^{\mathrm{w}} C_{k-1}\right) \mathbf{s}_{k-1}+C_{k} \mathbf{h}_{k-1}+\left(D_{k} \vec{H}_{c, k}-A_{k-1}^{\mathrm{w}} \vec{H}_{c, k-1}\right) \\
& +C_{k} \boldsymbol{\epsilon}_{k-1}+D_{k} \mathbf{e}_{k}-A_{k-1}^{\mathrm{w}} D_{k-1} \mathbf{e}_{k-1}+\mathbf{n}_{k}-A_{k-1}^{\mathrm{w}} \mathbf{n}_{k-1}
\end{aligned}
$$


The equations (9), (10), and (18) are now the state and the measurement equations for the case when $\mathbf{w}_{k} \neq 0$. The states are $\left[\mathbf{s}_{k}^{T}, \mathbf{w}_{k}^{T}\right]^{T}$, the output is $\widetilde{\mathbf{p}}_{k}$, the state noise is $\boldsymbol{\epsilon}_{k-1}$, and the observation noise is $C_{k} \epsilon_{k-1}+D_{k} \mathbf{e}_{k}-A_{k-1}^{\mathrm{w}} D_{k-1} \mathbf{e}_{k-1}+\mathbf{n}_{k}-A_{k-1}^{\mathrm{w}} \mathbf{n}_{k-1}$. The Kalman filter design procedure for this case can be found in most textbooks and will not be repeated here.

\section{B. Convergence of the Cooperative Kalman Filter}

Kalman filters converge if the time-varying system dynamics are uniformly completely controllable and uniformly completely observable [22]. In our case, these conditions are determined by the number of platforms employed, the geometric shape of the platform formation, and the speed of each platform. We develop a set of constraints for these factors so that the uniformly complete controllability and observability conditions are satisfied, which then guarantees convergence of the cooperative Kalman filter.

Let $\Phi(k, j)$ be the state transition matrix from time $t_{j}$ to $t_{k}$ where $k>j$, then one must have $\Phi(k, j)=A_{k-1}^{\mathrm{s}} A_{k-2}^{\mathrm{s}} \cdots A_{j}^{\mathrm{s}}$ and $\Phi(j, k)=\Phi^{-1}(k, j)$. The following lemma follows from direct calculation.

Lemma 3.1: For $\Phi(k, j)$ as defined above and $C_{k}$ as defined in (4), we have, for $k \neq j$, $\Phi(k, j)=\left[\begin{array}{cc}1 & \left(\mathbf{r}_{\mathrm{c}, k}-\mathbf{r}_{\mathrm{c}, j}\right)^{T} \\ 0 & I_{2 \times 2}\end{array}\right]$ and $C_{k} \Phi(k, j)=\left[\begin{array}{cc}1 & \left(\mathbf{r}_{1, k}-\mathbf{r}_{\mathrm{c}, j}\right)^{T} \\ \vdots & \vdots \\ 1 & \left(\mathbf{r}_{N, k}-\mathbf{r}_{\mathrm{c}, j}\right)^{T}\end{array}\right]$.

Remark 3.2: Note that this lemma holds for both $k>j$ and $k<j$. It applies to formations with any shape and any motion.

For clarity, we restate the definitions for uniformly complete controllability and uniformly complete observability in [22] using notations in this paper.

Definition 3.3: The state dynamics (9) are uniformly completely controllable if there exist $\tau_{1}>0, \beta_{1}>0$, and $\beta_{2}>0$ (independent of $k$ ) such that the controllability Grammian $\mathcal{C}(k, k-$ $\left.\tau_{1}\right)=\sum_{j=k-\tau_{1}}^{k} \Phi(k, j) M_{j-1} \Phi^{T}(k, j)$ satisfies $\beta_{1} I_{3 \times 3} \leq \mathcal{C}\left(k, k-\tau_{1}\right) \leq \beta_{2} I_{3 \times 3}$ for all $k>\tau_{1}$. Here $M_{j-1}$ is the covariance matrix for state noise $\boldsymbol{\epsilon}_{j-1}$.

Definition 3.4: Suppose $\mathbf{w}_{k}=0$ for all $k$. The state dynamics (9) together with the measurement equation (7) is uniformly completely observable if there exist $\tau_{2}>0, \beta_{3}>0$, and $\beta_{4}>0$ (independent of $k$ ) such that the observability Grammian $\mathcal{J}\left(k, k-\tau_{2}\right)=\sum_{j=k-\tau_{2}}^{k} \Phi^{T}(j, k) C_{j}^{T}\left[D_{j} U_{j} D_{j}^{T}+\right.$ $\left.R_{j}\right]^{-1} C_{j} \Phi(j, k)$ satisfies $\beta_{3} I_{3 \times 3} \leq \mathcal{J}\left(k, k-\tau_{2}\right) \leq \beta_{4} I_{3 \times 3}$ for all $k>\tau_{2}$. Here $U_{j}$ and $R_{j}$ are 
covariance matrices for noises $\mathbf{e}_{j}$ and $\mathbf{n}_{j}$ respectively.

Note that in these definitions, the " $\leq$ " is a relation between two symmetric matrices such that $A_{1} \leq A_{2}$ if and only if $\mathbf{x}^{T} A_{1} \mathbf{x} \leq \mathbf{x}^{T} A_{2} \mathbf{x}$ for any vector $\mathbf{x}$ with compatible dimension.

If $\mathbf{w}_{k} \neq 0$, the measurement equation is (18) instead of (7). Then the observability Grammian is $\mathcal{J}^{\mathrm{w}}\left(k, k-\tau_{2}\right)=\sum_{j=k-\tau_{2}}^{k} \Phi^{T}(j, k) \widetilde{C}_{j}^{T} \widetilde{R}_{j}^{-1} \widetilde{C}_{j} \Phi(j, k)$ where $\widetilde{C}_{j}=C_{j} A_{j-1}^{\mathrm{s}}-A_{j-1}^{\mathrm{w}} C_{j-1}$ and

$$
\widetilde{R}_{j}=C_{j} M_{j-1} C_{j}^{T}+D_{j} U_{j} D_{j}^{T}+A_{j-1}^{\mathrm{w}} D_{j-1} U_{j-1} D_{j-1} A_{j-1}^{\mathrm{w} T}+R_{j}+A_{j-1}^{\mathrm{w}} R_{j-1} A_{j-1}^{\mathrm{w} T} .
$$

The condition for uniformly complete observability is that for all $k>\tau_{2}, \beta_{3} I_{3 \times 3} \leq \mathcal{J}^{\mathrm{w}}(k, k-$ $\left.\tau_{2}\right) \leq \beta_{4} I_{3 \times 3}$.

In the following discussions, we derive constraints on the formations so that the uniformly complete controllability and observability conditions are satisfied by showing that there exist positive real numbers $\beta_{1}, \beta_{2}, \ldots, \beta_{28}$ that serve as time-independent bounds for various quantities. The actual value for these bounds do not affect the correctness of our arguments.

For uniformly complete controllability the following lemma holds.

Lemma 3.5: The state dynamics (9) are uniformly completely controllable if the following conditions are satisfied:

(Cd1) The symmetric matrix $M_{j-1}$ is uniformly bounded, i.e., $\beta_{5} I \leq M_{j-1} \leq \beta_{6} I$ for all $j$ and for some constants $\beta_{5}, \beta_{6}>0$.

(Cd2) The speed of each platform is uniformly bounded, i.e., $\left\|\mathbf{r}_{i, j}-\mathbf{r}_{i, j-1}\right\| \leq \beta_{7}$ for all time $j$, for $i=1, \ldots, N$, and for some constant $\beta_{7}>0$.

Proof: Due to condition (Cd1), the controllability Grammian satisfies $\beta_{5} \sum_{j=k-\tau_{1}}^{k} \Phi(k, j) \Phi^{T}(k, j) \leq$ $\mathcal{C}\left(k, k-\tau_{1}\right)$ and $\mathcal{C}\left(k, k-\tau_{1}\right) \leq \beta_{6} \sum_{j=k-\tau_{1}}^{k} \Phi(k, j) \Phi^{T}(k, j)$ for any $k$ and $\tau_{1}$ such that $k>\tau_{1}$. We first observe that $\Phi(k, j) \Phi^{T}(k, j)$ is a positive semi-definite symmetric matrix for each $j$ such that $k-\tau_{1} \leq j \leq k$. If we can find uniform bounds for each of these matrices i.e. $\Phi(k, j) \Phi^{T}(k, j)$, we obtain an overall bound for the controllability Grammian.

We apply Lemma 3.1 to compute $\Phi(k, j) \Phi^{T}(k, j)$, i.e.,

$$
\Phi(k, j) \Phi^{T}(k, j)=\left[\begin{array}{cc}
1+\|\delta \mathbf{r}(k, j)\|^{2} & (\delta \mathbf{r}(k, j))^{T} \\
\delta \mathbf{r}(k, j) & I_{2 \times 2}
\end{array}\right]
$$

where we define $\delta \mathbf{r}(k, j)=\mathbf{r}_{c, k}-\mathbf{r}_{c, j}$. The minimum eigenvalue of matrix (20) is

$$
\lambda_{\min }=\frac{1}{2}\left(\|\delta \mathbf{r}(k, j)\|^{2}+2-\sqrt{\left(\|\delta \mathbf{r}(k, j)\|^{2}+2\right)^{2}-4}\right)
$$


and the maximum eigenvalue is

$$
\lambda_{\max }=\frac{1}{2}\left(\|\delta \mathbf{r}(k, j)\|^{2}+2+\sqrt{\left(\|\delta \mathbf{r}(k, j)\|^{2}+2\right)^{2}-4}\right) .
$$

Since $(\mathrm{Cd} 2)$ is satisfied and $\delta \mathbf{r}(k, j)$ is the averaged movement over all platforms between time $j$ and $k$, we must have $\|\delta \mathbf{r}(k, j)\| \leq(k-j) \beta_{7} \leq \tau_{1} \beta_{7}$ for all $j \in\left[k-\tau_{1}, k\right]$. It is straightforward to show that $\lambda_{\min }$ assumes its minimum value when $\|\delta \mathbf{r}(k, j)\|=\tau_{1} \beta_{7}$. This minimum value is $\beta_{8}=\frac{1}{2}\left(\left(\tau_{1} \beta_{7}\right)^{2}+2-\sqrt{\left.\left(\tau_{1} \beta_{7}\right)^{2}+2\right)^{2}-4}\right)$. We can see that $\beta_{8}>0$. On the other hand, $\lambda_{\max }$ assumes its maximum value also when $\|\delta \mathbf{r}(k, j)\|=\tau_{1} \beta_{7}$. This maximum value is $\beta_{9}=\frac{1}{2}\left(\left(\tau_{1} \beta_{7}\right)^{2}+2+\sqrt{\left.\left(\tau_{1} \beta_{7}\right)^{2}+2\right)^{2}-4}\right)$, and $\beta_{9}>0$. Therefore, we conclude that $\beta_{8} I_{3 \times 3} \leq \Phi(k, j) \Phi^{T}(k, j) \leq \beta_{9} I_{3 \times 3}$ for all $j \in\left[k-\tau_{1}, k\right]$. Thus $\beta_{5} \tau_{1} \beta_{8} I_{3 \times 3} \leq \mathcal{C}\left(k, k-\tau_{1}\right) \leq$ $\beta_{6} \tau_{1} \beta_{9} I_{3 \times 3}$. Let $\beta_{1}=\beta_{5} \tau_{1} \beta_{8}$ and $\beta_{2}=\beta_{6} \tau_{1} \beta_{9}$. Since $\beta_{1}$ and $\beta_{2}$ do not depend on $k$, we have proved the uniformly complete controllability claim using Definition 3.3.

By the arguments for proving Lemma 3.5, we have also proved the following lemma.

Lemma 3.6: Suppose condition $(\mathrm{Cd} 2)$ is satisfied. Then there exist constants $\tau_{1}>0, \beta_{8}>0$, and $\beta_{9}>0$ such that the state transition matrices satisfy $\beta_{8} I_{3 \times 3} \leq \Phi(i, j) \Phi^{T}(i, j) \leq \beta_{9} I_{3 \times 3}$ for all $i, j \in\left[k-\tau_{1}, k\right]$ and for all $k>\tau_{1}$.

To prove uniformly complete observability, we also need an elementary lemma for which we do not show the proof.

Lemma 3.7: Suppose two $2 \times 1$ vectors $\mathbf{a}_{1}$ and $\mathbf{a}_{2}$ form an angle $\gamma$ such that $0<\gamma<\pi$. Then the minimum eigenvalue $\lambda_{\min }$ of the $2 \times 2$ matrix $A=\mathbf{a}_{1} \mathbf{a}_{1}^{T}+\mathbf{a}_{2} \mathbf{a}_{2}^{T}$ is strictly positive, i.e., $\lambda_{\min }>0$.

We have the following lemma regarding uniformly complete observability of a moving formation.

Lemma 3.8: Suppose $\mathbf{w}_{k}=0$ for all $k$. The state dynamics (9) with the measurement equation (7) are uniformly completely observable if $(\mathrm{Cd} 2)$ and the following conditions are satisfied:

(Cd3) The symmetric matrices $R_{j}$ and $U_{j}$ are uniformly bounded, i.e., $\beta_{10} I_{N \times N} \leq R_{j} \leq \beta_{11} I_{N \times N}$ and $0 \leq U_{j} \leq \beta_{12} I_{N \times N}$ for all $j$ and for some constants $\beta_{10}, \beta_{11}, \beta_{12}>0$.

(Cd4) The distance between each platform and the formation center is uniformly bounded from both above and below, i.e., $\beta_{13} \leq\left\|\mathbf{r}_{i, j}-\mathbf{r}_{c, j}\right\| \leq \beta_{14}$ for all $j$, for $i=1,2, \ldots, N$, and for some constants $\beta_{13}, \beta_{14}>0$. 
(Cd5) There exists a constant time difference $\tau_{2}$ and for all $k>\tau_{2}$, there exist time instances $j_{1}, j_{2} \in\left[k-\tau_{2}, k\right]$ where $j_{1}<j_{2}$, as well as two platforms indexed by $i_{1}$ and $i_{2}$, such that one of the following two conditions is satisfied:

(Cd5.1) The two vectors, $\mathbf{r}_{i_{1}, j_{1}}-\mathbf{r}_{c, j_{1}}$ and $\mathbf{r}_{c, j_{1}}-\mathbf{r}_{c, j_{2}}$ form an angle $\gamma_{1}$ that is uniformly bounded away from 0 or $\pi$. In other words, there exists a positive constant $\beta_{15}<1$ such that $\sin \left(\gamma_{1} / 2\right) \geq \beta_{15}$.

(Cd5.2) The two vectors, $\mathbf{r}_{i_{1}, j_{1}}-\mathbf{r}_{c, j_{1}}$ and $\mathbf{r}_{i_{2}, j_{2}}-\mathbf{r}_{c, j_{2}}$ form an angle $\gamma_{2}$ that is uniformly bounded away from 0 or $\pi$. In other words, there exists a positive constant $\beta_{15}<1$ such that $\sin \left(\gamma_{2} / 2\right) \geq \beta_{15}$.

Proof: Condition (Cd3) implies that $U_{j}$ is positive semi-definite, and condition (Cd4) implies that every component of $D_{j}$ is bounded above. Hence the matrix $D_{j} U_{j} D_{j}^{T}$ is a positive semidefinite matrix with its maximum eigenvalue bounded above. Also from $(\mathrm{Cd} 3), R_{j}$ is a positive definite symmetric matrix. Therefore, Weyl's theorem (c.f. [47], Theorem 4.3.1) that states the eigenvalues of the sum of two Hermitian matrices are bounded above by the sum of the two maximum eigenvalues and bounded below by the sum of the two minimum eigenvalues can be applied to $R_{j}+D_{j} U_{j} D_{j}^{T}$. This implies that there exist positive constants $\beta_{16}, \beta_{17}>0$ such that $\beta_{16} I_{N \times N} \leq\left(R_{j}+D_{j} U_{j} D_{j}^{T}\right) \leq \beta_{17} I_{N \times N}$ where $\beta_{16} \geq \beta_{10}$ and $\beta_{17} \geq \beta_{11}$. Thus, one must have $\beta_{17}^{-1} \sum_{j=k-\tau_{2}}^{k} \Phi^{T}(j, k) C_{j}^{T} C_{j} \Phi(j, k) \leq \mathcal{J}\left(k, k-\tau_{2}\right) \leq \beta_{16}^{-1} \sum_{j=k-\tau_{2}}^{k} \Phi^{T}(j, k) C_{j}^{T} C_{j} \Phi(j, k)$ for all $k>\tau_{2}$. Next, we prove the existence of positive uniform upper and lower bounds for $\sum_{j=k-\tau_{2}}^{k} \Phi^{T}(j, k) C_{j}^{T} C_{j} \Phi(j, k)$ for all $k>\tau_{2}$.

First for the upper bound, according to Lemma 3.1, we can compute

$$
\Phi^{T}(j, k) C_{j}^{T} C_{j} \Phi(j, k)=\left[\begin{array}{cc}
N & \left(\mathbf{r}_{c, j}-\mathbf{r}_{c, k}\right)^{T} \\
\left(\mathbf{r}_{c, j}-\mathbf{r}_{c, k}\right) & \sum_{i=1}^{N}\left(\mathbf{r}_{i, j}-\mathbf{r}_{c, k}\right)\left(\mathbf{r}_{i, j}-\mathbf{r}_{c, k}\right)^{T}
\end{array}\right] .
$$

The conditions $(\mathrm{Cd} 2)$ and $(\mathrm{Cd} 4)$ imply that each component of the above matrix is bounded above. Hence there exists $\beta_{18}>0$ such that $\Phi^{T}(j, k) C_{j}^{T} C_{j} \Phi(j, k) \leq \beta_{18} I_{3 \times 3}$.

We now use condition $(\mathrm{Cd} 5)$ to argue that there exists the lower bound $\beta_{19}>0$ such that $\beta_{19} I_{3 \times 3} \leq \sum_{j=k-\tau_{2}}^{k} \Phi^{T}(j, k) C_{j}^{T} C_{j} \Phi(j, k)$. Consider the two time instances indexed by $j_{1}$ and $j_{2}$ as given by condition (Cd5). It is sufficient to show that the matrix $\mathcal{I}$ defined by $\mathcal{I}=$ $\Phi^{T}\left(j_{1}, k\right) C_{j_{1}}^{T} C_{j_{1}} \Phi\left(j_{1}, k\right)+\Phi^{T}\left(j_{2}, k\right) C_{j_{2}}^{T} C_{j_{2}} \Phi\left(j_{2}, k\right)$ satisfies $\mathcal{I} \geq \beta_{19} I_{3 \times 3}$. 
Because $\Phi\left(j_{1}, k\right)=\Phi\left(j_{1}, j_{2}\right) \Phi\left(j_{2}, k\right)$, we have $\mathcal{I}=\Phi^{T}\left(j_{1}, k\right) \mathcal{I}_{1} \Phi^{T}\left(j_{1}, k\right)$ where

$$
\mathcal{I}_{1}=\Phi^{T}\left(j_{1}, j_{2}\right) C_{j_{1}}^{T} C_{j_{1}} \Phi\left(j_{1}, j_{2}\right)+C_{j_{2}}^{T} C_{j_{2}} .
$$

By direct calculation one can verify that

$$
C_{j_{2}}^{T} C_{j_{2}}=\left[\begin{array}{cc}
N & 0 \\
0 & \sum_{i=1}^{N}\left(\mathbf{r}_{i, j_{2}}-\mathbf{r}_{c, j_{2}}\right)\left(\mathbf{r}_{i, j_{2}}-\mathbf{r}_{c, j_{2}}\right)^{T}
\end{array}\right] .
$$

Using Lemma 3.1 and the fact that $\sum_{i=1}^{N}\left(\mathbf{r}_{i, j_{1}}-\mathbf{r}_{c, j_{1}}\right)=0$, we have

$$
\begin{aligned}
\Phi^{T}\left(j_{1}, j_{2}\right) C_{j_{1}}^{T} C_{j_{1}} \Phi\left(j_{1}, j_{2}\right) & =\left[\begin{array}{cc}
1 & \left(\mathbf{r}_{c, j_{1}}-\mathbf{r}_{c, j_{2}}\right)^{T} \\
\mathbf{r}_{c, j_{1}}-\mathbf{r}_{c, j_{2}} & \left(\mathbf{r}_{c, j_{1}}-\mathbf{r}_{c, j_{2}}\right)\left(\mathbf{r}_{c, j_{1}}-\mathbf{r}_{c, j_{2}}\right)^{T}
\end{array}\right] \\
& +\left[\begin{array}{cc}
N-1 & 0 \\
0 & \sum_{i=1}^{N}\left(\mathbf{r}_{i, j_{1}}-\mathbf{r}_{c, j_{1}}\right)\left(\mathbf{r}_{i, j_{1}}-\mathbf{r}_{c, j_{1}}\right)^{T}
\end{array}\right] \\
& +\left[\begin{array}{cc}
0 & 0 \\
0 & (N-1)\left(\mathbf{r}_{c, j_{1}}-\mathbf{r}_{c, j_{2}}\right)\left(\mathbf{r}_{c, j_{1}}-\mathbf{r}_{c, j_{2}}\right)^{T}
\end{array}\right] .
\end{aligned}
$$

Then the matrix $\mathcal{I}_{1}$ can be obtained by adding (23) and (24) together. Considering the platforms $i_{1}$ and $i_{2}$ in $(\mathrm{Cd} 5.1)$ and $(\mathrm{Cd} 5.2)$, we can further decompose $\mathcal{I}_{1}$ as the sum of two matrices: $\mathcal{I}_{1}=\mathcal{I}_{2}+\mathcal{I}_{3}$ where $\mathcal{I}_{2}=\left[\begin{array}{cc}1 & 0 \\ 0 & \mathcal{I}_{4}\end{array}\right]$ with

$$
\mathcal{I}_{4}=\left(\mathbf{r}_{i_{1}, j_{1}}-\mathbf{r}_{c, j_{1}}\right)\left(\mathbf{r}_{i_{1}, j_{1}}-\mathbf{r}_{c, j_{1}}\right)^{T}+\left(\mathbf{r}_{i_{1}, j_{2}}-\mathbf{r}_{c, j_{2}}\right)\left(\mathbf{r}_{i_{1}, j_{2}}-\mathbf{r}_{c, j_{2}}\right)^{T}+\left(\mathbf{r}_{c, j_{1}}-\mathbf{r}_{c, j_{2}}\right)\left(\mathbf{r}_{c, j_{1}}-\mathbf{r}_{c, j_{2}}\right)^{T} \text {, }
$$

and $\mathcal{I}_{3}$ is a positive semi-definite matrix.

Because either condition (Cd5.1) or condition (Cd5.2) is satisfied, according to Lemma 3.7, there exists $\beta_{21}>0$ such that the matrix $\mathcal{I}_{4} \geq \beta_{21} I_{2 \times 2}$. Therefore, using the Weyl's theorem (c.f. [47], Theorem 4.3.1) we conclude that there exists $\beta_{20}>0$ such that $\mathcal{I}_{1} \geq \beta_{20} I_{3 \times 3}$. Then Lemma 3.6 guarantees the existence of $\beta_{19}>0$ such that $\mathcal{I} \geq \beta_{19} I_{3 \times 3}$, which further implies that $\mathcal{J}\left(k, k-\tau_{2}\right) \geq \beta_{19} I_{3 \times 3}$.

Because both the uniform upper and lower bounds for the observability Grammian $\mathcal{J}\left(k, k-\tau_{2}\right)$ exist for all $k>\tau_{2}$, we have proved the uniformly complete observability claim.

Remark 3.9: We do not need to give formulas for all the $\beta$ s in conditions (Cd1)-(Cd5). The values for these $\beta \mathrm{s}$ will not change the fact that the filter converges, but only affect the speed of convergence. 
We now consider the case when the colored noise $\mathbf{w}_{k} \neq 0$. The following lemma establishes the sufficient conditions for uniformly complete observability.

Lemma 3.10: The state dynamics (9) and (10) with the measurement equation (18) are uniformly completely observable if $(\mathrm{Cd} 2),(\mathrm{Cd} 4)$, and the following conditions are satisfied:

(Cd6) The symmetric matrix $\widetilde{R}_{j}$ is uniformly bounded i.e. $\beta_{22} I_{N \times N} \leq \widetilde{R}_{j} \leq \beta_{23} I_{N \times N}$ for all $j$ and some positive constants $\beta_{22}$ and $\beta_{23}$.

(Cd7) The matrix $A_{j-1}^{\mathrm{w}}$ and the matrix $C_{j-1}$ satisfy $\beta_{24} I_{N \times N} \leq\left(I_{N \times N}-A_{j-1}^{\mathrm{w}}\right)^{T}\left(I_{N \times N}-A_{j-1}^{\mathrm{w}}\right)^{T} \leq$ $\beta_{25} I_{N \times N}$ and $\beta_{26} I_{N \times N} \leq C_{j-1}^{T} C_{j-1} \leq \beta_{27} I_{N \times N}$ for some positive constants $\beta_{24}, \beta_{25}, \beta_{26}$ and $\beta_{27}$.

(Cd8) The constants $\beta_{7}$ in (Cd2) and the constants $\beta_{24}, \beta_{26}$ in (Cd7) satisfy $\beta_{7} \sqrt{N}+\beta_{28}<$ $\sqrt{\beta_{24} \beta_{26}}$ for some positive constant $\beta_{28}$.

Proof: Condition (Cd6) implies that $\beta_{23}^{-1} \sum_{j=k-\tau_{2}}^{k} \Phi^{T}(j, k) \widetilde{C}_{j}^{T} \widetilde{C}_{j} \Phi^{T}(j, k) \leq \mathcal{J}^{\mathrm{w}}\left(k, k-\tau_{2}\right)$ and $\mathcal{J}^{\mathrm{w}}\left(k, k-\tau_{2}\right) \leq \beta_{22}^{-1} \sum_{j=k-\tau_{2}}^{k} \Phi^{T}(j, k) \widetilde{C}_{j}^{T} \widetilde{C}_{j} \Phi^{T}(j, k)$.

Consider $\widetilde{C}_{j}=C_{j} A_{j-1}^{\mathrm{s}}-A_{j-1}^{\mathrm{w}} C_{j-1}$. Using Lemma 3.1 we have $C_{j} A_{j-1}^{\mathrm{s}}=C_{j-1}+\delta C_{j}$ where $\delta C_{j}=\left[\begin{array}{cc}0 & \left(\mathbf{r}_{1, j}-\mathbf{r}_{\mathrm{c}, j-1}\right)^{T} \\ \vdots & \vdots \\ 0 & \left(\mathbf{r}_{N, j}-\mathbf{r}_{\mathrm{c}, j-1}\right)^{T}\end{array}\right]$. Therefore, $\widetilde{C}_{j}=\left(I_{N \times N}-A_{j-1}^{\mathrm{w}}\right) C_{j-1}+\delta C_{j}$. Applying the Hoffman-Wielandt theorem ( [47], Theorem 7.3.8), we have

$$
\left|\sqrt{\lambda_{\min }\left(\widetilde{C}_{j}^{T} \widetilde{C}_{j}\right)}-\sqrt{\lambda_{\min }\left(C_{j-1}^{T}\left(I_{N \times N}-A_{j-1}^{\mathrm{w}}\right)^{T}\left(I_{N \times N}-A_{j-1}^{\mathrm{w}}\right) C_{j-1}\right)}\right| \leq \sqrt{\operatorname{trace}\left(\delta C_{j} \delta C_{j}^{T}\right)} .
$$

Thus using condition (Cd8), we have

$$
\begin{aligned}
\sqrt{\lambda_{\min }\left(\widetilde{C}_{j}^{T} \widetilde{C}_{j}\right)} & \geq \sqrt{\lambda_{\min }\left(C_{j-1}^{T}\left(I_{N \times N}-A_{j-1}^{\mathrm{w}}\right)^{T}\left(I_{N \times N}-A_{j-1}^{\mathrm{w}}\right) C_{j-1}\right)}-\operatorname{trace}\left(\delta C_{j} \delta C_{j}^{T}\right) \\
& \geq \sqrt{\beta_{24} \beta_{26}}-\beta_{7} \sqrt{N}>\beta_{28} .
\end{aligned}
$$

Therefore $\sum_{j=k-\tau_{2}}^{k} \Phi^{T}(j, k) \widetilde{C}_{j}^{T} \widetilde{C}_{j} \Phi^{T}(j, k)$ is uniformly bounded below, away from singular matrices. It is also uniformly bounded above by conditions $(\mathrm{Cd} 2),(\mathrm{Cd} 4)$ and $(\mathrm{Cd} 7)$. Hence $\mathcal{J}^{\mathrm{w}}\left(k, k-\tau_{2}\right)$ is uniformly bounded below, away from singular matrices, and above.

With Lemmas 3.5, 3.8, and 3.10 justified, Theorem 7.4 in [22] can be applied to prove the convergence of the cooperative Kalman filter.

Theorem 3.11: (Theorem 7.4 in [22]) Consider the time-varying linear system formed by the state equation (9) and (10) with the measurement equation (18). If the system is uniformly 
completely controllable and uniformly completely observable, then the Kalman filter for this system converges.

The following theorems can be viewed as corollaries of Theorem 3.11.

Theorem 3.12: Suppose $\mathbf{w}_{k}=0$ for all $k$. Consider the state dynamics (9) with the measurement equation (7). If the conditions (Cd1)-(Cd5) are satisfied, then the cooperative Kalman filter given by (13)-(17) converges and the error covariance matrix $P_{k}$ is bounded as $k \rightarrow \infty$.

Theorem 3.13: Consider the state dynamics (9) and (10) with the measurement equation (18). If the conditions $(\mathrm{Cd} 1)-(\mathrm{Cd} 2),(\mathrm{Cd} 4)$ and $(\mathrm{Cd} 6)-(\mathrm{Cd} 8)$ are satisfied, then the cooperative Kalman filter for this case converges and the error covariance matrix is bounded as $k \rightarrow \infty$.

\section{Formation design principles}

The conditions $(\mathrm{Cd} 1)-(\mathrm{Cd} 8)$ have provided us the following intuitive guidelines for formation design to yield successful cooperative Kalman filters.

1) If $N \geq 3$, there is no penalty in fixing the orientation of the formation, as long as the shape is nonsingular. A singular formation occurs when all platforms are on a straight line or collapse to a point. In fact, if the formation is singular only occasionally, the Kalman filter will still converge.

2) If $N=2$ or a line formation is desired, then one should make the orientation of the line change over time, such as in a rocking or rolling motion.

3) The speed of the platforms needs to be bounded from both above and below to guarantee the controllability and observability conditions at the same time. Such bounds depend on the strength of the error covariance matrices.

4) In case of a correlated field, the relation between the size of the formation and the speed of the formation should satisfy (Cd8).

\section{The cross formation and steady state error covariance}

As an example, we select a fixed coordinate frame formed by unit vectors $\mathbf{e}_{1}$ and $\mathbf{e}_{2}$ and arrange the four platforms in a symmetric formation as shown in Fig. 1 so that

1) $\mathbf{r}_{2, k}-\mathbf{r}_{1, k}$ is perpendicular to $\mathbf{r}_{3, k}-\mathbf{r}_{4, k}$,

2) $\left\|\mathbf{r}_{2, k}-\mathbf{r}_{\mathrm{c}, k}\right\|=\left\|\mathbf{r}_{\mathrm{c}, k}-\mathbf{r}_{1, k}\right\|=a_{k}$ and $\left\|\mathbf{r}_{3, k}-\mathbf{r}_{\mathrm{c}, k}\right\|=\left\|\mathbf{r}_{\mathrm{c}, k}-\mathbf{r}_{4, k}\right\|=b_{k}$ and

3) the $\mathbf{e}_{1}$ vector is aligned with $\mathbf{r}_{2, k}-\mathbf{r}_{1, k}$ and the $\mathbf{e}_{2}$ vector is aligned with $\mathbf{r}_{3, k}-\mathbf{r}_{4, k}$. 
Then, in the lab coordinate frame,

$$
C_{k}=\left[\begin{array}{ccc}
1 & -a_{k} & 0 \\
1 & a_{k} & 0 \\
1 & 0 & b_{k} \\
1 & 0 & -b_{k}
\end{array}\right] D_{k}=\left[\begin{array}{cccc}
a_{k}^{2} & 0 & 0 & 0 \\
a_{k}^{2} & 0 & 0 & 0 \\
0 & 0 & 0 & b_{k}^{2} \\
0 & 0 & 0 & b_{k}^{2}
\end{array}\right]
$$

which have very simple form because of the symmetry.

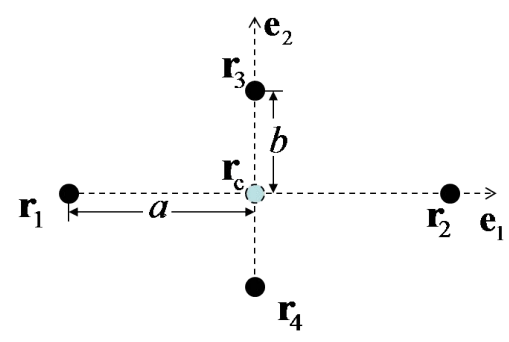

Fig. 1. A symmetric arrangement of a formation of four sensor platforms. We design $a_{k}$ and $b_{k}$ to minimize the mean square error when constant Kalman gain is adopted.

We may design the steady state formation shape so that the steady state error covariance of the cooperative Kalman filter is minimized. In the case when $\mathbf{w}_{k}=0$ for all $k$, the error covariance matrix satisfies the Riccati equation:

$$
P_{\infty}^{-1}=\left[A_{\infty}^{\mathrm{s}} P_{\infty}\left(A_{\infty}^{\mathrm{s}}\right)^{T}+M_{\infty}\right]^{-1}+C_{\infty}^{T}\left[D_{\infty} U_{\infty} D_{\infty}^{T}+R_{\infty}\right]^{-1} C_{\infty}
$$

The mission goal at steady state is to move the formation along a level curve. Then consider equation (8): as $k \rightarrow \infty$, since $\left(\mathbf{r}_{c, k}-\mathbf{r}_{c, k-1}\right)^{T} \nabla z\left(\mathbf{r}_{c, k-1}\right)=0$, we can replace $A_{\infty}^{\text {s }}$ by $I_{3 \times 3}$ in equation (29) as $k \rightarrow \infty$

We then determine the steady state values of $a_{k}$ and $b_{k}$ that minimize the error covariance. Suppose $a_{k} \rightarrow a$ and $b_{k} \rightarrow b$ as $k \rightarrow \infty$. The following proposition holds.

Proposition 3.14: Suppose as $k \rightarrow \infty, M_{k} \rightarrow \sigma_{3}^{2} I_{3 \times 3}, R_{k} \rightarrow \sigma_{1}^{2} I_{4 \times 4}$, and $U_{k} \rightarrow \sigma_{2}^{2} I_{4 \times 4}$. Then $P_{k(+)}$ converges to a diagonal matrix $P_{\infty}$. The trace of $P_{\infty}$ is

$$
\operatorname{tr}\left(P_{\infty}\right)=-\frac{3}{2} \sigma_{3}^{2}+\frac{1}{2} \sum_{i=1}^{3} \sqrt{\sigma_{3}^{4}+\frac{4 \sigma_{3}^{2}}{c_{i}}}
$$

where

$$
c_{1}=\frac{2}{2 \sigma_{2}^{2} a^{4}+\sigma_{1}^{2}}+\frac{2}{2 \sigma_{2}^{2} b^{4}+\sigma_{1}^{2}}, \quad c_{2}=\frac{2 a^{2}}{\sigma_{1}^{2}}, \text { and } c_{3}=\frac{2 b^{2}}{\sigma_{1}^{2}} \text {. }
$$


Proof: By direct calculation, one can verify that, as $k \rightarrow \infty$,

$$
C_{k}^{T}\left[D_{k} U_{k} D_{k}^{T}+R_{k}\right]^{-1} C_{k} \rightarrow \operatorname{diag}\left\{\frac{2}{2 \sigma_{2}^{2} a^{4}+\sigma_{1}^{2}}+\frac{2}{2 \sigma_{2}^{2} b^{4}+\sigma_{1}^{2}}, \frac{2 a^{2}}{\sigma_{1}^{2}}, \frac{2 b^{2}}{\sigma_{1}^{2}}\right\}=\operatorname{diag}\left\{c_{1}, c_{2}, c_{3}\right\} .
$$

As $k \rightarrow \infty, A_{k-1}^{\mathrm{s}}$ is replaced by $I_{3 \times 3}$. Therefore, $P_{\infty}$ solved from (29) is a diagonal matrix. By direct calculation, one can verify that the trace of $P_{\infty}$ is given by (30).

Using the cross formation has simplified the procedure in solving the steady state Riccati equation (29). The resulting $P_{\infty}$ satisfies $\operatorname{tr}\left(P_{\infty}\right) \geq 0$; a minimum of $\operatorname{tr}\left(P_{\infty}\right)$ can be found when $a$ and $b$ assume value between $(0, \infty)$. Finding analytical solutions for the optimal values $a$ and $b$ that minimize $\operatorname{tr}\left(P_{\infty}\right)$ requires solving a 4-th order algebraic equation, which is best solved using numerical methods. Since there are only two variables to optimize, the problem is rudimentary for most numeric packages.

We can then compute $P_{\infty}, C_{\infty}$, and $D_{\infty}$. In the case that the noise covariance $M_{k}, U_{k}$ and $R_{k}$ are time-invariant, these computation can be performed "off-line", i.e., before any observations are made. Hence, a Kalman filter gain $K_{\infty}$ can be given beforehand as $K_{\infty}=P_{\infty} C_{\infty}^{T}\left[C_{\infty} P_{\infty} C_{\infty}^{T}+\right.$ $\left.D_{\infty} U_{\infty} D_{\infty}^{T}+R_{\infty}\right]^{-1}$.

\section{E. Adaptive formation}

The constant Kalman gain $K_{\infty}$ computed beforehand is based on the assumptions on the steady state noise covariance. Therefore, the steady state gain may only be suboptimal if the noise covariances are time varying. If the constant gain $K_{\infty}$ is used, the formation shape may be adjusted to achieve optimal Kalman filtering. Furthermore, if the regular Kalman filter gain $K_{k}$ is used, we show the error covariance of the estimates can be minimized by adjusting the shape of the platform formation e.g. changing $a$ and $b$. Our method extends the adaptive scheme previously developed in [1] to minimize estimation error based on instantaneous measurements.

It is well known that sub-optimal filters can be derived using $K_{\infty}$. For example, when $\mathbf{w}_{k}=0$, the update equation can be $\mathbf{s}_{k(+)}=\mathbf{s}_{k(-)}+K_{\infty}\left(\mathbf{p}_{k}-C_{k} \mathbf{s}_{k(-)}-D_{k} \vec{H}_{\mathrm{c}, k}\right)$. A unique property of using multiple mobile sensor platforms is that we can adjust $C_{k}$ and $D_{k}$, by adjusting the geometric shape of the formation, to minimize estimation error for this sub-optimal filter. The resulting formation is then adaptive and the resulting filter is optimal.

Another well-known result for Kalman filter design indicates that the error function to be minimized at step $k$ is $J_{k}=\frac{1}{2} \operatorname{tr}\left[\left(I_{3 \times 3}-K_{k} C_{k}\right) P_{k(-)}\left(I_{3 \times 3}-K_{k} C_{k}\right)^{T}+K_{k}\left(D_{k} U_{k} D_{k}^{T}+R_{k}\right) K_{k}^{T}\right]$. 
In fact, if $C_{k}$ and $D_{k}$ are known, the gain $K_{k}$ that minimizes $J_{k}$ is exactly the Kalman filter gain.

This formation can be made adaptive when the regular Kalman gain $K_{k}$ is used. This is because by equation (15), since $P_{k(-)}$ is known, $K_{k}$ is a function of $C_{k}$ and $D_{k}$. The resulting adaptive formation and the estimates will minimize the cost function $J_{k}$ at each step $k$. Therefore, we modify step (3) in Subsection III-A:

(3A) Let $\widehat{K}_{k}$ be a function of $C_{k}$ and $D_{k}$ i.e. $\widehat{K}_{k}\left(C_{k}, D_{k}\right)=P_{k(-)} C_{k}^{T}\left[C_{k} P_{k(-)} C_{k}^{T}+D_{k} U_{k} D_{k}^{T}+\right.$ $\left.R_{k}\right]^{-1}$. Find $C_{k}^{*}$ and $D_{k}^{*}$ so that the function $J_{k}=\frac{1}{2} \operatorname{tr}\left[\left(I_{3 \times 3}-\widehat{K}_{k} C_{k}\right) P_{k(-)}\left(I_{3 \times 3}-\widehat{K}_{k} C_{k}\right)^{T}+\right.$ $\left.\widehat{K}_{k}\left(D_{k} U_{k} D_{k}^{T}+R_{k}\right) \widehat{K}_{k}^{T}\right]$ is minimized. Then let $K_{k}=\widehat{K}_{k}\left(C_{k}^{*}, D_{k}^{*}\right)$.

This new step is generally difficult to compute since $C_{k}$ and $D_{k}$ are matrices. But as we have discussed, using symmetric formations will greatly reduce the complexity.

\section{F. Section Summary}

The convergence of the cooperative Kalman filter algorithms imposes constraints on feasible platform formations, and the shape of the formation affects error in the filters. In order for the cooperative Kalman filters to converge, formations should be designed to make the filter systems uniformly completely controllable and uniformly completely observable. Formations with $N=2$ may need to rotate, but formations with $N \geq 3$ can have fixed orientation if the formation is not co-linear. We also note that the formations can be adaptively adjusted on-line to minimize estimation error. Symmetric formations help to reduce complexity in theoretical analysis, computation, and operation.

\section{Cooperative Estimation of the Hessian}

An estimate of the Hessian, $H_{\mathrm{c}, k}$, is needed to enable the Kalman filter. At the end of the $(k-1)$ th time step, we have obtained an estimate $\mathbf{s}_{k-1(+)}$ from the cooperative Kalman filter. This includes an estimate $z_{c, k-1}$ for $z\left(\mathbf{r}_{\mathrm{c}, k-1}\right)$ and an estimate $\mathbf{d}_{\mathrm{c}, k-1}$ for $\nabla z\left(\mathbf{r}_{\mathrm{c}, k-1}\right)$. We outline the procedure to cooperatively compute $H_{\mathrm{c}, k}$ as follows:

1) Start with an estimate or an initial guess $H_{\mathrm{c}, k-1}$.

2) Use a one-step filter to reduce noise in the new measurements.

3) Compute $H_{c, k}$. 


\section{A. A Cooperative One-step Filter to Reduce Noise}

Using the computed estimates $\mathbf{s}_{k-1(+)}$ and $\vec{H}_{\mathrm{c}, k-1}$, we can make predictions, before the arrival of measurements at time step $k$, for the field value at the positions of the sensor platforms that have moved from $\mathbf{r}_{i, k-1}$ to $\mathbf{r}_{i, k}$ as $\mathbf{z}_{k}^{\mathrm{P}}=C_{k} \mathbf{s}_{k-1(+)}+D_{k} \vec{H}_{\mathrm{c}, k-1}$. The error of the prediction $\mathbf{z}_{k}^{\mathrm{P}}$ compared to the true value $\mathbf{z}_{k}$ is Gaussian i.e. $\mathbf{z}_{k}^{\mathrm{P}}=\mathbf{z}_{k}+\boldsymbol{\psi}_{k}$. From properties of the Kalman filter, the covariance of $\boldsymbol{\psi}_{k}$ is $G_{k}=C_{k} P_{k-1(+)}^{\mathrm{s}} C_{k}^{T}$ where $P_{k-1(+)}^{\mathrm{s}}$ is the error covariance in the estimate $\mathbf{s}_{k-1(+)}$.

We then take new measurements at the $k$ th step using all platforms. Let $\mathbf{p}_{k}$ be the vector of the measurements and $\mathbf{z}_{k}^{\mathrm{P}}$ be the vector of the predictions. Let the updated measurements $\widehat{\mathbf{z}}_{k}$ be

$$
\widehat{\mathbf{z}}_{k}=\left(I+G_{k}\left(W_{k}+R_{k}\right)^{-1}\right)^{-1} \mathbf{z}_{k}^{\mathrm{P}}+\left(I+\left(R_{k}+W_{k}\right) G_{k}^{-1}\right)^{-1} \mathbf{p}_{k} .
$$

Such $\widehat{\mathbf{z}}_{k}$ minimizes the cost function

$$
J_{k}=\frac{1}{2}\left[\left(\widehat{\mathbf{z}}_{k}-\mathbf{z}_{k}^{\mathrm{P}}\right)^{T} G_{k}^{-1}\left(\widehat{\mathbf{z}}_{k}-\mathbf{z}_{k}^{\mathrm{P}}\right)+\left(\mathbf{p}_{k}-\widehat{\mathbf{z}}_{k}\right)\left(W_{k}+R_{k}\right)^{-1}\left(\mathbf{p}_{k}-\widehat{\mathbf{z}}_{k}\right)\right] .
$$

As we can see, $G_{k}$ serves as the weighting matrix that balances using the information from previous estimates and from current measurements. The following proposition has been proved in our previous work [48].

Proposition 4.1: The estimator given in equation (33) is unbiased with error covariance matrix $\left(I+G_{k}\left(W_{k}+R_{k}\right)^{-1}\right)^{-1} G_{k}$.

\section{B. Cooperative Estimation of Hessian}

Using the cooperative Kalman filter, we may obtain a prediction for the $\mathbf{s}_{k}$ as

$$
\mathbf{s}_{k(-)}=A_{k-1}^{\mathrm{s}} \mathbf{s}_{k-1(+)}+\mathbf{h}_{k-1} .
$$

Using the one-step filter we also have the filtered measurements $\widehat{\mathbf{z}}_{k}$. If the number of the sensor

platforms $N \geq 3$ and the formation is not co-linear, then since $\widehat{\mathbf{z}}_{k}=C_{k} \mathbf{s}_{k(-)}+D_{k} \vec{H}_{k}$, one may conjecture that the Hessian estimate can be solved, using the least mean square method, as $\vec{H}_{k}=\left(D_{k}^{T} D_{k}\right)^{-1} D_{k}^{T}\left(\widehat{\mathbf{z}}_{k}-C_{k} \mathbf{s}_{k(-)}\right)$. However, it can be shown that for $N \leq 4$, the matrix $D_{k}^{T} D_{k}$ is singular, hence the least mean square method will not work.

We now introduce an alternative method to estimate the Hessian that utilizes the relationship between the Hessian and the curvature of level curves. 
1) Curvature and Hessian: The level curve passing through the center of the formation $\mathbf{r}_{\mathrm{c}}$ can be parametrized by its arc-length $s$, hence $z(\mathbf{r}(s))$ is constant for all values of $s$. Suppose the gradient $\nabla z$ does not vanish along the curve. The unit normal vector to the level curve is defined as $\mathbf{y}_{1}(s)=\frac{\nabla z(\mathbf{r}(s))}{\|\nabla z(\mathbf{r}(s))\|}$, and at any given point, the unit tangent vector to the curve, denoted by $\mathbf{x}_{1}(s)$, satisfies $\mathbf{x}_{1}(s) \cdot \mathbf{y}_{1}(s)=0$. Then we have the following Frenet-Serret equations [49]:

$$
\frac{d \mathbf{x}_{1}(s)}{d s}=\kappa(s) \mathbf{y}_{1}(s) \text { and } \frac{d \mathbf{y}_{1}(s)}{d s}=-\kappa(s) \mathbf{x}_{1}(s),
$$

where $\kappa(s)$ is defined as the Frenet-Serret curvature of the level curve.

With this configuration, because $\nabla z\left(\mathbf{r}_{\mathrm{c}}\right) \cdot \mathbf{x}_{1}=0$ along the level curve, the derivative with respect to $s$ is $\frac{d}{d s} \nabla z\left(\mathbf{r}_{\mathrm{c}}\right) \cdot \mathbf{x}_{1}+\nabla z\left(\mathbf{r}_{\mathrm{c}}\right) \cdot \frac{d \mathbf{x}_{1}}{d s}=0$ which implies $\mathbf{x}_{1}^{T} \nabla^{2} z\left(\mathbf{r}_{\mathrm{c}}\right) \mathbf{x}_{1}+\left\|\nabla z\left(\mathbf{r}_{\mathrm{c}}\right)\right\| \mathbf{y}_{1}$. $\kappa(s) \mathbf{y}_{1}=0$ where $\nabla^{2} z\left(\mathbf{r}_{\mathrm{c}}\right)$ is the Hessian of $z$ at $\mathbf{r}_{\mathrm{c}}$. Because $\mathbf{x}_{1}$ is the unit vector along the $\mathbf{x}_{1}$-axis, in the Frenet-Serret frame we have $\partial_{\mathrm{xx}} z\left(\mathbf{r}_{\mathrm{c}}\right)+\left\|\nabla z\left(\mathbf{r}_{\mathrm{c}}\right)\right\| \kappa(s)=0$. This suggests that we can obtain $H_{\mathrm{xx}, k}$, the estimate for $\partial_{\mathrm{xx}} z\left(\mathrm{r}_{\mathrm{c}, k}\right)$, by

$$
H_{\mathrm{xx}, k}=-\left\|\mathbf{d}_{\mathrm{c}, k}\right\| \kappa_{\mathrm{c}, k}
$$

where $\mathbf{d}_{\mathrm{c}, k}$ is the estimate for the gradient $\nabla z\left(\mathbf{r}_{\mathrm{c}, k}\right)$ and $\kappa_{\mathrm{c}, k}$ is the estimate for the curvature $\kappa\left(\mathbf{r}_{\mathrm{c}, k}\right)$.

On the other hand, we have $\nabla z\left(\mathbf{r}_{\mathrm{c}}\right) \cdot \mathbf{y}_{1}=\left\|\nabla z\left(\mathbf{r}_{\mathrm{c}}\right)\right\|$. Taking derivatives on both sides of this equation with respect to $s$, we get $\mathbf{x}_{1}^{T} \nabla^{2} z\left(\mathbf{r}_{\mathrm{c}}\right) \mathbf{y}_{1}-\left\|\nabla z\left(\mathbf{r}_{\mathrm{c}}\right)\right\| \mathbf{y}_{1} \cdot \kappa(s) \mathbf{x}_{1}=\frac{d}{d s}\left\|\nabla z_{\mathrm{c}}\right\|$. This implies that $\partial_{\mathrm{xy}} z\left(\mathbf{r}_{\mathrm{c}}\right)=\frac{d}{d s}\left\|\nabla z_{\mathrm{c}}\right\|$. Therefore, the estimate $H_{\mathrm{xy}, k}$ for $\partial_{\mathrm{xy}} z\left(\mathbf{r}_{\mathrm{c}, k}\right)$ is

$$
H_{\mathrm{xy}, k}=\frac{d}{d s}\left\|\mathbf{d}_{\mathrm{c}, k}\right\| .
$$

The estimates $H_{\mathrm{xx}, k}$ and $H_{\mathrm{xy}, k}$ are elements of $H_{\mathrm{c}, k}$ in the Frenet-Serret coordinate system. Since the field $z(\cdot)$ is smooth, we require $H_{\mathrm{yx}, k}=H_{\mathrm{xy}, k}$. We also need to find out $H_{\mathrm{yy}, k}$ to determine $H_{\mathrm{c}, k}$.

2) Algorithm to estimate the Hessian: We show how to use four sensor platforms to estimate the Hessian at $\mathbf{r}_{\mathrm{c}, k}$. For $N>4$, the algorithm can be used directly. For $N<4$, the algorithm can be extended by combining measurements from different time instances.

Since the procedure only involves information for step $k$, we drop the subscript $k$ in this section for simplicity.

With a formation of four moving sensor platforms, we are able to estimate $\kappa(s)$ for the level curve at the center of the formation by the following steps: 


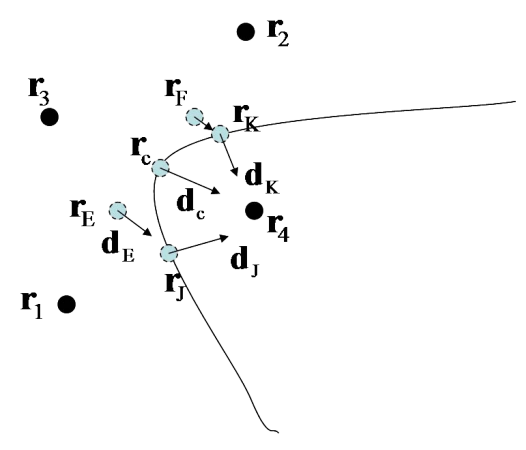

Fig. 2. Detection of a level curve using four sensor platforms. $\mathbf{r}_{\mathrm{c}}$ denotes the center of the entire formation. $\mathbf{r}_{\mathrm{E}}$ denotes the center of the formation formed by $\mathbf{r}_{1}, \mathbf{r}_{3}$ and $\mathbf{r}_{4} . \mathbf{r}_{\mathrm{F}}$ denotes the center of the formation formed by $\mathbf{r}_{2}, \mathbf{r}_{3}$ and $\mathbf{r}_{4} . \mathbf{r}_{\mathrm{J}}$ and $\mathbf{r}_{\mathrm{K}}$ are located on the same level curve as $\mathbf{r}_{\mathrm{c}}$.

(s.1) Compute an estimate of the field value and gradient at the center $\mathbf{r}_{\mathrm{c}}$ using (34).

(s.2) Considering the formation defined by $\mathbf{r}_{1}, \mathbf{r}_{3}$ and $\mathbf{r}_{4}$, obtain the estimates $z_{\mathrm{E}}$ and $\mathbf{d}_{\mathrm{E}}$ at the center $\mathbf{r}_{\mathrm{E}}$ of this three platform formation (Fig. 2) by solving the following equations for $i=1,3,4: \widehat{z}_{i}=z_{\mathrm{E}}+\mathbf{d}_{\mathrm{E}} \cdot\left(\mathbf{r}_{i}-\mathbf{r}_{\mathrm{E}}\right)+\frac{1}{2}\left(\mathbf{r}_{i}-\mathbf{r}_{\mathrm{E}}\right)^{T} H^{\mathrm{P}}\left(\mathbf{r}_{i}-\mathbf{r}_{\mathrm{E}}\right)$ where the $\widehat{z}_{i}$ are given by (33) and $H^{\mathrm{P}}$ is the estimate of Hessian taken from previous time step. Let $\widehat{\mathbf{z}}_{\mathrm{E}}=\left[\widehat{z}_{1}, \widehat{z}_{3}, \widehat{z}_{4}\right]^{T}$, $\mathbf{s}_{\mathrm{E}}=\left[z_{\mathrm{E}}, \mathbf{d}_{\mathrm{E}}^{T}\right]^{T}$, and $C_{\mathrm{E}}=\left[\begin{array}{cc}1 & \left(\mathbf{r}_{1}-\mathbf{r}_{\mathrm{E}}\right)^{T} \\ 1 & \left(\mathbf{r}_{3}-\mathbf{r}_{\mathrm{E}}\right)^{T} \\ 1 & \left(\mathbf{r}_{4}-\mathbf{r}_{\mathrm{E}}\right)^{T}\end{array}\right]$. Let $D_{\mathrm{E}}$ be the $4 \times 3$ matrix with its three row vectors given by $\frac{1}{2}\left(\left(\mathbf{r}_{i}-\mathbf{r}_{\mathrm{E}}\right) \otimes\left(\mathbf{r}_{i}-\mathbf{r}_{\mathrm{E}}\right)\right)^{T}$ for $i=1,3,4$. Then $\widehat{\mathbf{z}}_{\mathrm{E}}=C_{E} \mathbf{S}_{\mathrm{E}}+D_{E} \vec{H}^{\mathrm{P}}$ which implies that $\mathbf{s}_{\mathrm{E}}=C_{\mathrm{E}}^{-1}\left(\widehat{\mathbf{z}}_{\mathrm{E}}-D_{E} \vec{H}^{\mathrm{P}}\right)$.

(s.3) Along the positive or negative direction of $\mathbf{d}_{\mathrm{E}}$, we may find the point $\mathbf{r}_{\mathrm{J}}$ (Fig. 2) where $z_{\mathrm{J}}=z_{\mathrm{c}}$ using $\mathbf{r}_{\mathrm{J}}=\mathbf{r}_{\mathrm{E}}+\left(z_{\mathrm{c}}-z_{\mathrm{E}}\right) \frac{\mathbf{d}_{\mathrm{E}}}{\left\|\mathbf{d}_{\mathrm{E}}\right\|}$.

(s.4) Estimate $\mathbf{d}_{\mathrm{J}, k}$ by solving the following equations for $i=1,3,4: \widehat{z}_{i}=z_{\mathrm{J}}+\mathbf{d}_{\mathrm{J}} \cdot\left(\mathbf{r}_{i}-\mathbf{r}_{\mathrm{J}}\right)+$ $\frac{1}{2}\left(\mathbf{r}_{i}-\mathbf{r}_{\mathrm{J}}\right)^{T} H^{\mathrm{P}}\left(\mathbf{r}_{i}-\mathbf{r}_{\mathrm{J}}\right)$.

(s.5) Repeat the steps (s.2), (s.3) and (s.4) for the formation consisting of $\mathbf{r}_{2}, \mathbf{r}_{3}$ and $\mathbf{r}_{4}$ with appropriate changes in the subscripts for points $\mathbf{r}_{\mathrm{F}}$ and $\mathbf{r}_{\mathrm{K}}$ (Fig. 2).

(s.6) Let $\mathbf{y}_{1 \mathrm{~J}}, \mathbf{y}_{1 \mathrm{~K}}$ and $\mathbf{y}_{1 \mathrm{c}}$ denote the unit vectors along the directions of the gradient $\mathbf{d}_{\mathrm{J}}, \mathbf{d}_{\mathrm{K}}$ and $\mathbf{d}_{\mathrm{c}}$. Define $\delta \theta_{\mathrm{L}}=\arccos \left(\mathbf{y}_{1 \mathrm{~J}} \cdot \mathbf{y}_{1 \mathrm{c}}\right), \delta s_{\mathrm{L}}=\left\|\mathbf{r}_{\mathrm{J}}-\mathbf{r}_{\mathrm{c}}\right\|, \delta \theta_{\mathrm{R}}=\arccos \left(\mathbf{y}_{1 \mathrm{~K}} \cdot \mathbf{y}_{1 \mathrm{c}}\right)$, and $\delta s_{\mathrm{R}}=\left\|\mathbf{r}_{\mathrm{K}}-\mathbf{r}_{\mathrm{c}}\right\|$. Obtain the estimate for $\kappa(s)$ at $\mathbf{r}_{\mathrm{c}}$ as $\kappa_{\mathrm{c}}=\frac{1}{2}\left(\frac{\delta \theta_{\mathrm{L}}}{\delta s_{\mathrm{L}}}+\frac{\delta \theta_{\mathrm{R}}}{\delta s_{\mathrm{R}}}\right)$. Obtain the estimate for $H_{\mathrm{xx}}$ according to (36). 
(s.7) Approximate $\frac{d}{d s}\left\|\nabla z_{\mathrm{c}}\right\|$ by $\frac{d}{d s}\left\|\nabla z_{\mathrm{c}}\right\|=\frac{\left\|\mathbf{d}_{\mathrm{K}}\right\|-\left\|\mathbf{d}_{\mathrm{J}}\right\|}{\delta s_{\mathrm{L}}+\delta s_{\mathrm{R}}}$. Then using (37), the estimate $H_{\mathrm{xy}}$ is $H_{\mathrm{xy}}=\frac{\left\|\mathbf{d}_{\mathrm{K}}\right\|-\left\|\mathbf{d}_{\mathrm{J}}\right\|}{\delta s_{\mathrm{L}}+\delta s_{\mathrm{R}}}$.

(s.8) Solve $\widehat{z}_{i}=z_{\mathrm{c}}+\mathbf{d}_{\mathrm{c}} \cdot\left(\mathbf{r}_{i}-\mathbf{r}_{\mathrm{c}}\right)+\frac{1}{2}\left(\mathbf{r}_{i}-\mathbf{r}_{\mathrm{c}}\right)^{T} H_{\mathrm{c}}\left(\mathbf{r}_{i}-\mathbf{r}_{\mathrm{c}}\right)$ for $H_{\mathrm{yy}}$ where $i=1,2,3,4$.

The resulting matrix $H_{\mathrm{c}, k}$ can be used directly as the estimate for the Hessian at the $k$ th step. Or we may repeat steps (s.1)-(s.8) starting from $H_{\mathrm{c}, k}$ to get a new estimate for the curvature and then to improve the estimate $H_{\mathrm{c}, k}$. The procedure becomes an iterative numerical algorithm that solves the set of nonlinear equations that $z_{c}, H_{\mathrm{xx}, k}, H_{\mathrm{xy}, k}$ and $H_{\mathrm{yy}, k}$ satisfy given $\widehat{z}_{i, k}$ for $i=1,2,3,4$. The prediction from step $k-1$ provides a reasonable initial value for this iterative algorithm.

\section{Formation Shape And Orientation Control}

In this section, we show that by using a powerful tool called the Jacobi transform, the formation shape and orientation dynamics can be decoupled from the dynamics of the center (or the centroid) of the formation. Therefore, a formation shape and orientation controller can be designed without considering the motion of the formation center, and such a controller will not affect the motion of the formation center. We can control the formation to have fixed orientation or to rotate according to prescribed angular speed around the formation center.

\section{A. Formation Control with Fixed Orientation}

We view the entire formation as a deformable body. The shape and orientation of this deformable body can be described using a special set of Jacobi vectors, c.f. [37], [38], [50]-[52] and the references therein. Here, assuming that all platforms have unit mass, we define the set of Jacobi vectors as $\mathbf{q}_{j}, j=1,2, \ldots, N-1$ satisfying $\left[\mathbf{r}_{\mathrm{c}}, \mathbf{q}_{1}, \ldots, \mathbf{q}_{N-1}\right]=\left[\mathbf{r}_{1}, \mathbf{r}_{2}, \ldots, \mathbf{r}_{N}\right] \Psi$ where $\Psi$ defines a linear coordinate transform that decouples the kinetic energy of the entire system, i.e., $K=\frac{1}{2} \sum_{i=1}^{N}\left\|\dot{\mathbf{r}}_{i}\right\|^{2}=\frac{1}{2}\left(N\left\|\dot{\mathbf{r}}_{\mathrm{c}}\right\|^{2}+\sum_{i=1}^{N-1}\left\|\dot{\mathbf{q}}_{i}\right\|^{2}\right)$. We call $\Psi$ the Jacobi transform. This allows us to separate motion of the center from shape and orientation changes. The transform $\Psi$ is guaranteed to exist. For example, when $N=4$, the following definition of Jacobi vectors may be used

$$
\mathbf{q}_{1}=\frac{1}{\sqrt{2}}\left(\mathbf{r}_{2}-\mathbf{r}_{1}\right), \quad \mathbf{q}_{2}=\frac{1}{\sqrt{2}}\left(\mathbf{r}_{3}-\mathbf{r}_{4}\right), \text { and } \mathbf{q}_{3}=\frac{1}{2}\left(\mathbf{r}_{4}+\mathbf{r}_{3}-\mathbf{r}_{2}-\mathbf{r}_{1}\right)
$$


Lagrange's equations for the formation in the lab frame are simply the set of Newton's equations: $\ddot{\mathbf{r}}_{i}=\mathbf{f}_{i}$ where $\mathbf{f}_{i}$ is the control force for the $i$ th platform for $i=1, \ldots, N$. In terms of the Jacobi vectors, these equations are equivalent to

$$
\ddot{\mathbf{q}}_{j}=\mathbf{u}_{j} \text { and } N \ddot{\mathbf{r}}_{\mathrm{c}}=\mathbf{f}_{\mathrm{c}}
$$

where $j=1, \ldots, N-1$, and $\mathbf{u}_{j}$ and $\mathbf{f}_{\mathrm{c}}$ are equivalent forces satisfying

$$
\left[\mathbf{f}_{\mathrm{c}}, \mathbf{u}_{1}, \ldots, \mathbf{u}_{N-1}\right]=\left[\mathbf{f}_{1}, \mathbf{f}_{2}, \ldots, \mathbf{f}_{N}\right] \Psi^{-T} .
$$

We design the control forces $\mathbf{u}_{i}, j=1,2, \ldots, N-1$, so that as $t \rightarrow \infty, \mathbf{q}_{j} \rightarrow \mathbf{q}_{j}^{0}$ where $\mathbf{q}_{j}^{0}$ are desired vectors that define a constant formation. For example, when $N=4$, we want

$$
\mathbf{q}_{1}(t) \rightarrow \frac{a}{\sqrt{2}} \mathbf{e}_{1}, \quad \mathbf{q}_{2}(t) \rightarrow-\frac{b}{\sqrt{2}} \mathbf{e}_{2}, \quad \text { and } \mathbf{q}_{3}(t) \rightarrow \mathbf{0}
$$

where $\mathbf{e}_{1}$ and $\mathbf{e}_{2}$ are the two unit vectors defining the lab coordinate frame in Section III-D, and $a$ and $b$ are the optimal values determined by methods in Section III-D to minimize the steady state error covariance. Since the controlled dynamics for $\mathbf{q}_{j}$ are linear, the following control laws guarantee the goal (41) with an exponential rate of convergence: $\mathbf{u}_{j}=-K_{2}\left(\mathbf{q}_{j}-\mathbf{q}_{j}^{0}\right)-K_{3} \dot{\mathbf{q}}_{j}$ where $K_{2}, K_{3}>0$ are constant gains. This control law design method can also by applied to stabilize the adaptive formation obtained in Section III-E with the assumption that the optimal formation will not change very fast over time.

Comparing to existing formation control and stability results for formation with fixed shape and orientation (for example, [53], [54]), this controller is much simpler and its stability is easy to prove. This is due to the fact that the reduced system is linear after the Jacobi transform.

\section{B. Formation Control with Rotation}

When only two sensor platforms are available, if we control the motion of the two platform formation such that the formation is rotating periodically, then the system will satisfy the observability condition and the Kalman filter will converge. The difference between controller design in this section and in the previous section is that the orientation of the platform formation will be changing.

The platform formation where there are $N$ platforms can be described by the Jacobi vectors $\mathbf{q}_{j}$ where $j=1,2, \ldots, N-1$. The orientation of the collection of the Jacobi vectors in the inertial frame can be described by an angle $\psi$ which is the angle between a selected Jacobi vector and 
the horizontal axis of the lab fixed frame. It does not matter which Jacobi vector to select. Let the matrix $g$ be $g=\left[\begin{array}{cc}\cos \psi & \sin \psi \\ -\sin \psi & \cos \psi\end{array}\right]$. We define vectors $\boldsymbol{\rho}_{j}$ as $\boldsymbol{\rho}_{j}=g^{-1} \mathbf{q}_{j}$. Let $\Omega$ be the angular velocity of the formation. Taking time derivatives on both sides of $\mathbf{q}_{j}=g \boldsymbol{\rho}_{j}$ yields $\dot{\mathbf{q}}_{j}=g\left(\dot{\boldsymbol{\rho}}_{j}+\Omega \times \boldsymbol{\rho}_{j}\right)$. Take time derivative again on both sides yields

$$
\mathbf{u}_{j}=g\left(\ddot{\boldsymbol{\rho}}_{j}+2 \Omega \times \dot{\boldsymbol{\rho}}_{j}+\Omega \times\left(\Omega \times \boldsymbol{\rho}_{j}\right)+\dot{\Omega} \times \boldsymbol{\rho}_{j}\right)
$$

We then design $\ddot{\boldsymbol{\rho}}_{j}$ and $\dot{\Omega}$ such that, for $j=1,2, \ldots, N-1$,

$$
\ddot{\boldsymbol{\rho}}_{j}=-K_{4}\left(\boldsymbol{\rho}_{j}-\boldsymbol{\rho}_{j}^{0}\right)-K_{5} \dot{\boldsymbol{\rho}}_{j} \text { and } \dot{\Omega}=-K_{6}\left(\Omega-\Omega_{0}\right)+\dot{\Omega}_{0}
$$

where $\boldsymbol{\rho}_{j}^{0}$ is a constant vector and $\Omega_{0}$ is a differentiable function of $t$. Then $\mathbf{u}_{j}$ can be computed using (42).

Once we have designed the combined force $f_{c}$ to control the center of the formation, then the control forces $\mathbf{f}_{i}, i=1,2, \ldots, N$ can be computed using (40). In Section VI, we design $\mathbf{f}_{\mathrm{c}}$ so that the center of the formation tracks a level curve. We note that our results in controller design for the platform formations pertain only to the deterministic formation dynamics; this certainty equivalence approach does not produce optimal controllers with input noise.

\section{FORMATION MOTION CONTROL}

In this section, we derive the equations governing a Newtonian particle moving in a scalar field. Then we design tracking control laws so that a particle can be controlled to follow any non-trivial level curve. The particle is identified with the center of the formation so that a level curve tracking behavior is achieved. Such level curve tracking behaviors complement gradient climbing behaviors in cooperative exploration strategies and help reveal structure in an unknown field.

\section{A. Particle Motion in a Scalar Field}

The center of the platform formation is modeled as a unit mass Newtonian particle with its position represented by $\mathbf{r}_{\mathrm{c}}$. The system equation for such particle is the Newton's equation $\ddot{\mathbf{r}}_{\mathrm{c}}=\mathbf{f}_{\mathrm{c}}$. Such equation can be written in an equivalent Frenet-Serret form which is more convenient for the tracking purpose [14], [15]. 
We define the speed of the particle as $\alpha$ and the acceleration $v_{\mathrm{c}}$ for $\alpha \neq 0$ as

$$
\alpha=\left\|\dot{\mathbf{r}}_{\mathrm{c}}\right\| \text { and } v_{\mathrm{c}}=\mathbf{f}_{\mathrm{c}} \cdot \frac{\dot{\mathbf{r}}_{\mathrm{c}}}{\alpha} .
$$

Then the equation for speed control is

$$
\dot{\alpha}=v_{\mathrm{c}}
$$

We let $v_{\mathrm{c}}=-K_{1}(\alpha-1)$. As time $t \rightarrow \infty, \alpha$ converges to unit speed exponentially with a rate determined by $K_{1}>0$.

We define a unit velocity vector $\mathbf{x}_{2}$ as $\mathbf{x}_{2}=\frac{\dot{\mathbf{r}}_{c}}{\alpha}$. We define a second unit vector $\mathbf{y}_{2}$ as the vector perpendicular to $\mathbf{x}_{2}$ that forms a right handed frame with $\mathbf{x}_{2}$ so that $\mathrm{x}_{2}$ and $\mathbf{y}_{2}$ lie in the plane of the page and the vector $\mathbf{x}_{2} \times \mathbf{y}_{2}$ points towards the reader. Then the steering control can be defined as $u_{\mathrm{c}}=\frac{1}{\alpha^{2}} \mathbf{f}_{\mathbf{c}} \cdot \mathbf{y}_{2}$. Using the facts that

$$
\mathbf{f}_{\mathrm{c}}=\left(\mathbf{f}_{\mathrm{c}} \cdot \mathbf{y}_{2}\right) \mathbf{y}_{2}+\left(\mathbf{f}_{\mathrm{c}} \cdot \mathbf{x}_{2}\right) \mathbf{x}_{2}
$$

and $\mathbf{x}_{2} \cdot \mathbf{y}_{2}=0$, we have the following equations:

$$
\dot{\mathbf{x}}_{2}=u_{\mathrm{c}} \alpha \mathbf{y}_{2} \text { and } \dot{\mathbf{y}}_{2}=-u_{\mathrm{c}} \alpha \mathbf{x}_{2}
$$

The equations (45) and (47) describe the particle motion in the Frenet-Serret form. Equation (46) shows the equivalence between Newton's equation and the Frenet-Serret form when $\alpha \neq 0$ : once the speed control $v_{\mathrm{c}}$ and the steering control $u_{\mathrm{c}}$ are determined, the total force $\mathbf{f}_{\mathrm{c}}$ can be determined.

Consider the smooth scalar field $z(\mathbf{r})$ in the plane. With the speed of the particle under control, we design a steering control for the particle so that it will track a level curve of $z(\cdot)$. The procedure can be found in our previous works [14], [15]. Here we briefly summarize and explain the results.

At any time instant $t$, there is a level curve of $z(\cdot)$ passing through $\mathbf{r}_{\mathrm{c}}$. At this position $\mathbf{r}_{\mathrm{c}}$, we let $\mathbf{y}_{1}$ be the unit vector in the direction of the gradient of the field $z(\cdot)$, and let $\mathbf{x}_{1}$ be the unit tangent vector to the level curve. By convention, $\mathbf{x}_{1}$ and $\mathbf{y}_{1}$ form a right handed coordinate frame with $\mathbf{x}_{1} \times \mathbf{y}_{1}$ pointing to the reader. This coordinate convention is identical to the one used in Section IV-B to derive equation (35).

For convenience, we introduce a variable $\theta \in(-\pi, \pi]$ such that $\cos \theta=\mathbf{x}_{1} \cdot \mathbf{x}_{2}$ and $\sin \theta=$ $-\mathbf{y}_{1} \cdot \mathbf{x}_{2}$. Along the trajectory of the center, it can be shown that $\dot{\theta}=\alpha\left(\kappa_{1} \cos \theta+\kappa_{2} \sin \theta-u_{c}\right)$ 
where $\kappa_{1}=-\frac{\mathbf{x}_{1}^{T} \nabla^{2} z \mathbf{x}_{1}}{\|\nabla z\|}, \kappa_{2}=\frac{\mathbf{x}_{1}^{T} \nabla^{2} z \mathbf{y}_{1}}{\|\nabla z\|}$, and $\nabla^{2} z$ represents the Hessian of the scalar field $z(\cdot)$. Meanwhile, along the trajectory of the center, the value of $z$ satisfies $\dot{z}=-\alpha\|\nabla z\| \sin \theta$.

\section{B. Steering Controller Design}

Suppose the scalar field has extrema $z_{\min }<z_{\max }$ which are allowed to be infinity. Let $\tilde{f}(z)$ be the derivative function of a function $\hbar(z)$ so that the following assumptions are satisfied:

(A1) $d \hbar / d z=\widetilde{f}(z)$, where $\tilde{f}(z)$ is a Lipschitz continuous function on $\left(z_{\min }, z_{\max }\right)$, and $\hbar(z)$ is continuously differentiable on $\left(z_{\min }, z_{\max }\right)$;

(A2) $\tilde{f}(C)=0$ and $\widetilde{f}(z) \neq 0$ if $z \neq C$;

(A3) $\lim _{z \rightarrow z_{\min }} \hbar(z)=\infty, \lim _{z \rightarrow z_{\max }} \hbar(z)=\infty$, and $\exists \widetilde{z}$ such that $\hbar(\widetilde{z})=0$.

We design the control law to be

$$
u_{\mathrm{c}}=\kappa_{1} \cos \theta+\kappa_{2} \sin \theta-2 \widetilde{f}(z)\|\nabla z\| \cos ^{2}\left(\frac{\theta}{2}\right)+K_{4} \sin \left(\frac{\theta}{2}\right)
$$

where $K_{4}>0$ is a constant control gain. We now assume that the speed of the center $\alpha=1$ is guaranteed by the speed controller $v_{c}$. Then the following proposition can be proved.

Proposition 6.1: Consider a smooth scalar field with bounded Hessian and bounded gradient that satisfies $\|\nabla z(\mathbf{r})\| \neq 0$ except for a finite number of points $\mathbf{r}_{\text {sup }}$ where $z\left(\mathbf{r}_{\text {sup }}\right)=z_{\min }$ or $z\left(\mathbf{r}_{\text {sup }}\right)=z_{\text {max }}$. Under the steering control law given in equation (48), we have $\theta \rightarrow 0$ and $z \rightarrow C$ asymptotically if the initial value $\theta\left(t_{0}\right) \neq \pi$ and $\mathbf{r}\left(t_{0}\right) \neq \mathbf{r}_{\text {sup }}$.

Proof: Let a Lyapunov candidate function be $V=-\log \cos ^{2}\left(\frac{\theta}{2}\right)+\hbar(z)$. Then its derivative can be shown as $\dot{V}=-\alpha K_{4} \frac{\sin ^{2} \frac{\theta}{2}}{\cos \frac{\theta}{2}}$. Therefore, if $\alpha>0$ we have $\dot{V} \leq 0$. The value of the Lyapunov function does not increase. Because our initial condition is such that $\cos \frac{\theta\left(t_{0}\right)}{2} \neq 0$, it is impossible for $\cos \frac{\theta(t)}{2}=0$ at any time instant $t$ since otherwise $V$ goes to infinity.

By the invariance theorem for non-autonomous systems ( [55], Theorem 4.4), we conclude that $\sin \frac{\theta}{2} \rightarrow 0$ as $t \rightarrow \infty$. $\theta(t)$ will not go to $\pi$ because we have shown that $\cos \frac{\theta(t)}{2} \neq 0$. This implies that $\theta(t) \rightarrow 0$ as $t \rightarrow \infty$.

On the other hand, note that $\left.\dot{\theta}\right|_{\sin \frac{\theta}{2}=0}=2 \alpha \widetilde{f}(z)\|\nabla z\|$. The right hand side is a uniformly continuous function of time since $z$ is constant and $\|\nabla z\|$ is a smooth function with bounded derivatives. Therefore, according to the Barbalat lemma, $\dot{\theta}$ must vanish as $t \rightarrow \infty$. This implies that either $\tilde{f}(z)=0$ or $\|\nabla z\|=0$. When $\|\nabla z\|=0$, we know $\mathbf{r}=\mathbf{r}_{\text {sup }}$. According to our assumption, $V$ goes to infinity at $\mathbf{r}=\mathbf{r}_{\text {sup }}$. Thus if we start with $\mathbf{r}\left(t_{0}\right) \neq \mathbf{r}_{\text {sup }}$, we must have 
$\|\nabla z\| \neq 0$ for all time $t>t_{0}$. Therefore, the only possibility left is $\tilde{f}(z)=0$ which implies that $z=C$.

\section{Simulation Results}

We first demonstrate the cooperative Kalman filtering and level curve tracking control using two sensor platforms. The potential field is generated by two identical positive charges in the plane with added correlated noise. It is desired that the two sensor platforms keep a distance of 1 unit length and rotate with a constant angular speed. Fig. 3 shows the snapshots of both platforms and the trajectory of the formation center when tracing a level curve. It can be observed that the trajectory of the center of the formation is smoother than the actual level curve with spatial noise.

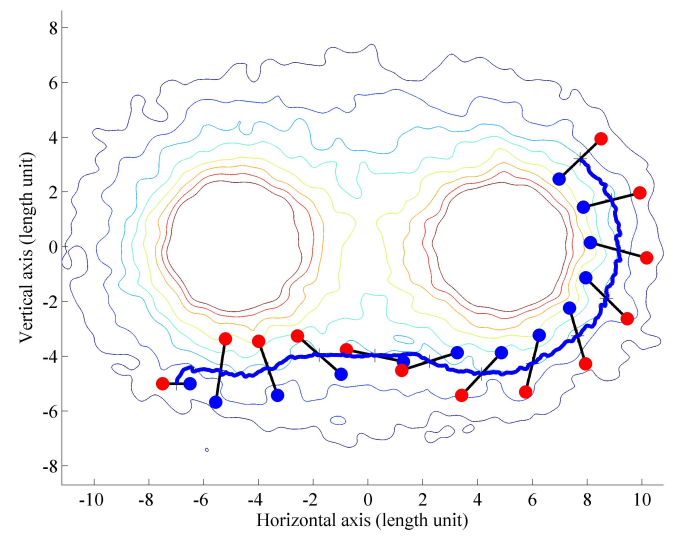

Fig. 3. Using two sensor platforms to track a level curve. The formation rotates at constant angular speed.

The level curve tracking algorithm is applicable to adaptive sampling using a mobile sensor network in the ocean. Adaptive ocean sampling is a central goal of our collaborative Adaptive Sampling and Prediction (ASAP) project [56]. The latest ASAP field experiment took place in August 2006 in Monterey Bay, California. Ten gliders were employed under continuous, automated coordinated control to collect maximally information-rich data for oceanographic research over the course of one month. The success of this field experiment sets a precedent for the usefulness of the kind of cooperative exploration represented by level curve tracking. This motivated our second illustration of level set tracking with four platforms in a model ocean field. 


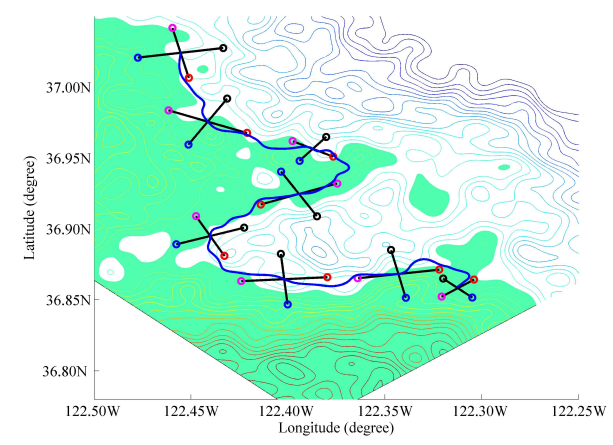

Fig. 4. Tracking, in simulation, the temperature level curve of $13^{\circ} \mathrm{C}$ in an estimated temperature field near Monterey Bay, CA on August 13, 2003. For visualization purposes, the level curve is accentuated. The trajectory of the center of formation is plotted with snapshots of the formation shown along the trajectory. The horizontal axis corresponds to longitude and the vertical axis to latitude.

In order to test our current algorithms on realistic ocean fields, we use a snapshot of the temperature field near Monterey Bay produced by the Harvard Ocean Prediction System (HOPS) [57]. This field reflects the temperature at 20 meters below sea surface on 00:00 GMT August 13th, 2003. This field was produced using remotely observed and in-situ data, including glider measurements during the Autonomous Ocean Sampling Network (AOSN) field experiment [18].

Four platforms are employed to track a level curve with temperature $13^{\circ} \mathrm{C}$. The trajectory of the formation center and the snapshots of the formation are plotted in Fig. 4. The center of the formation is controlled to travel at $1 \mathrm{~km}$ per hour. The orientation of the formation is adjusted so that the line connecting platforms 1 and 2 is aligned with the desired level curve. The shape of the formation is adjusted to minimize the Kalman filter error covariance. This can be observed from Fig. 5 where we plot the half distance between vehicles 1 and 2, i.e. the shape variable $a$, versus time. Fig. 6 shows the estimates of the temperature at the center of the formation versus time. One can see that the estimates center around $13^{\circ} \mathrm{C}$ with small error.

\section{SUMMARY AND FutURE WORK}

We have developed a cooperative Kalman filter that combines measurements from a small number of mobile sensor platforms to cooperatively explore a static planar scalar field. We show that the combined estimates satisfy an information dynamic model that does not depend on motion models of the platforms. Based on this model, we have rigorously justified a set 


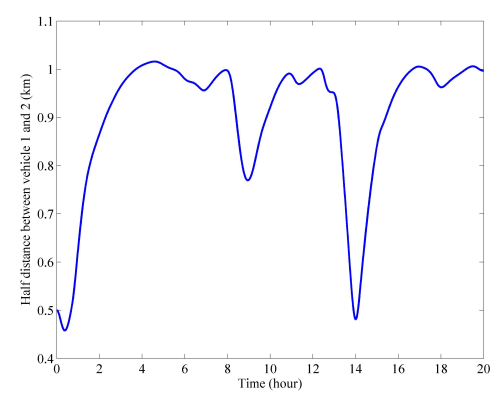

Fig. 5. The half distance between platforms 1 and 2, i.e. the shape variable a, versus time.

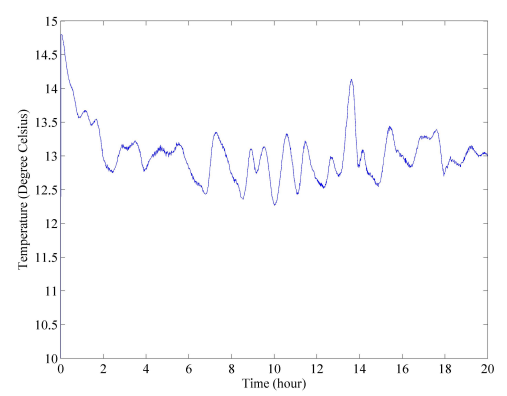

Fig. 6. The estimate $z_{\mathrm{c}}\left({ }^{\circ} \mathrm{C}\right)$ versus time (hour).

of sufficient conditions that guarantee the convergence of the cooperative Kalman filter. These sufficient conditions provide guidelines on mission design. We show how to adapt the formation shape to minimize error in the estimates. An algorithm has also been designed to estimate the local Hessian, which enables the Kalman filter and provides curvature estimates for steering control.

We take a geometric approach in formation control where reduction is performed on the total configuration space of the formation with the help of Jacobi vectors. The desired formation shape, orientation and motion can be stabilized using simple controllers with the help of the reduction method and the Jacobi transform. Both the filter and the formation shape controller are general for any number of platforms and arbitrary formations as long as conditions for uniform controllability and observability are satisfied. The filter and formation shape controller are combined with a steering control law for the center of the formation to perform level curve tracking behavior with provable convergence. 
There are open questions regarding the current results. The error covariance of $U_{k}$ and $M_{k}$ are not theoretically characterized and rely on heuristics and simulations, and we have only considered time-invariant fields in the plane. Hence, our ongoing work includes addressing the limitations, extending these methods to fast, time-varying scalar fields in three-dimensional space, and demonstrating the methods in experiments involving robotic mobile sensor platforms.

\section{REFERENCES}

[1] P. Ogren, E. Fiorelli, and N. E. Leonard, "Cooperative control of mobile sensor networks: Adaptive gradient climbing in a distributed environment," IEEE Transactions on Automatic Control, vol. 49, no. 8, pp. 1292-1302, 2004.

[2] D. Marthaler and A. L. Bertozzi, "Tracking environmental level sets with autonomous vehicles," in Recent Developments in Cooperative Control and Optimization, S. Butenko, R. Murphey, and P. Pardalos, Eds. Kluwer Academic Publishers, 2003.

[3] A. L. Bertozzi, M. Kemp, and D. Marthaler, "Determining environmental boundaries: Asynchronous communication and physical scales," in Cooperative Control, A Post-Workshop Volume: 2003 Block Island Workshop on Cooperative Control, V. Kumar, N. Leonard, and A. Morse, Eds. Springer, 2005, pp. 35-42.

[4] C. H. Hsieh, Z. Jin, D. Marthaler, B. Q. Nguyen, D. J. Tung, A. L. Bertozzi, and R. M. Murray, "Experimental validation of an algorithm for cooperative boundary tracking," in Proceedings of the American Control Conf., Portland, OR, 2005, pp. 1078-1083.

[5] D. Zarzhitsky, D. F. Spears, and W. M. Spears, "Swarms for chemical plume tracing," in Proc. of 2005 IEEE Symposium on Swarm Intelligence, Pasadena, California, 2005, pp. 249-256.

[6] F. Zhang, E. Justh, and P. S. Krishnaprasad, "Boundary following using gyroscopic control," in Proc. of 43rd IEEE Conf. on Decision and Control, Atlantis, Paradise Island, Bahamas, 2004, pp. 5204-5209.

[7] J. Clark and R. Fierro, "Cooperative hybrid control of robotic sensors for perimeter detection and tracking," in Proc. 2006 American Control Conf., Portland, OR, June 6-10, 2005, pp. 3500-3505.

[8] M. A. Hsieh and V. Kumar, "Pattern generation with multiple robots," Proc. 2006 IEEE International Conf. on Robotics and Automation, pp. 2442-2447, 2006.

[9] S. Susca, S. Martínez, and F. Bullo, "Monitoring environmental boundaries with a robotic sensor network," in Proc. American Control Conf. 2006, Minneapolis, MN, 2006, pp. 2072-2077.

[10] S. B. Andersson and J. Park, "Tip steering for fast imaging in AFM," in Proc. 2005 American Control Conf., Portland, OR, June 6-10, 2005, pp. 2469-2474.

[11] F. Zhang and N. Leonard, "Generating contour plots using multiple sensor platforms," in Proc. of 2005 IEEE Symposium on Swarm Intelligence, Pasadena, California, 2005, pp. 309-314.

[12] S. Kalantar and U. Zimmer, "Control of open contour formations of autonomous underwater vehicles," International Journal of Advanced Robotics Systems, vol. 2, no. 4, pp. 309-316, 2005.

[13] J. Cortés, S. Martínez, T. Karatas, and F. Bullo, "Coverage control for mobile sensing networks," IEEE Transactions on Robotics and Automation, vol. 20, no. 2, pp. 243-255, 2004.

[14] F. Zhang and N. E. Leonard, "Coordinated patterns of unit speed particles on a closed curve," Systems and Control Letters, vol. 56, no. 6, pp. 397-407, 2007. 
[15] F. Zhang, D. M. Fratantoni, D. Paley, J. Lund, and N. E. Leonard, "Control of coordinated patterns for ocean sampling," International Journal of Control, vol. 80, no. 7, pp. 1186-1199, 2007.

[16] G. J. Pottie and W. J. Kaiser, "Wireless integrated network sensors," Communications of the ACM, vol. 43, no. 5, pp. 51-58, 2000.

[17] D. Culler, D. Estrin, and M. Srivastava, "Overview of sensor networks," IEEE Computer Magazine, vol. 37, no. 8, pp. 41-49, 2004.

[18] N. E. Leonard, D. Paley, F. Lekien, R. Sepulchre, D. Fratantoni, and R. Davis, "Collective motion, sensor networks and ocean sampling," Proceedings of IEEE, vol. 95, no. 1, pp. 48-74, 2007.

[19] S. Martínez and F. Bullo, "Optimal sensor placement and motion coordination for target tracking," Automatica, vol. 42, no. 4, pp. 661-668, 2006.

[20] R. E. Kalman, "When is a linear control system optimal?" ASME Transactions, Journal of Basic Engineering, vol. 86, pp. 51-60, 1964.

[21] R. S. Bucy, "Global theory of the Riccati equation," Journal of Computer and System Sciences, vol. 1, no. 7, pp. 349-361, 1967.

[22] A. H. Jazwinski, Stochastic Processes and Filtering Theory. Academic Press, New York, 1970.

[23] W. W. Hager and L. L. Horowitz, "Convergence and stability properties of the discrete Riccati equation and the associated control and filtering problems," SIAM J. Control and Optimization, vol. 14, pp. 295-312, 1976.

[24] B. D. O. Anderson and J. Moore, "Detectability and stabilizability of time-varying discrete-time linear systems," SIAM Journal on Control and Optimization, vol. 19, no. 1, pp. 20-32, 1981.

[25] S. W. Chan, G. C. Goodwin, and K. S. Sin, "Convergence properties of the Riccati difference equation in optimal filtering of nonstabilizable systems," IEEE Transactions on Automatic Control, vol. 29, no. 2, pp. 110-118, 1984.

[26] R. Olfati-Saber, "Distributed Kalman filter with embedded consensus filters," in Proc. of 44th IEEE Conf. Decision and Control and 2005 European Control Conference (CDC-ECC '05), Seville, Spain, 2005, pp. 8179-8184.

[27] R. Olfati-Saber and J. S. Shamma, "Consensus filters for sensor networks and distributed sensor fusion," in Proc. of 44th IEEE Conf. Decision and Control and 2005 European Control Conference (CDC-ECC '05), Seville, Spain, 2005, pp. 6698-6703.

[28] P. Yang, R. A. Freeman, and K. M. Lynch, "Distributed cooperative active sensing using consensus filters," in Proc. of 2007 IEEE International Conference on Robotics and Automation, Rome, Italy, 2007, pp. 405-410.

[29] I. Hussein, "A Kalman filter-based control strategy for dynamic coverage control," in Proc. of 2007 American Control Conf., New York, New York, 2007, pp. 3721-3726.

[30] S. Martínez, "Distributed representation of spatial fields through an adaptive interpolation scheme," in Proc. of 2007 American Control Conf., New York, New York, 2007, pp. 2750-2755.

[31] A. Jadbabaie, J. Lin, and A. S. Morse, "Coordination of groups of mobile agents using nearest neighbor rules," IEEE Trans. on Automatic Control, vol. 48, no. 6, pp. 988-1001, 2003.

[32] Z. Lin, M. Broucke, and B. Francis, "Local control strategies for groups of mobile autonomous agents," IEEE Transactions on Automatic Control, vol. 49, no. 4, pp. 622-629, 2004.

[33] R. Olfati-Saber and R. M. Murray, "Consensus problems in networks of agents with switching topology and time-delays," IEEE Trans. on Automatic Control, vol. 49, no. 9, pp. 1520-1533, 2004.

[34] L. Moreau, "Stability of multiagent systems with time-dependent communication links," IEEE Transactions on Automatic Control, vol. 50, no. 2, pp. 169-182, 2005. 
[35] W. Ren and R. W. Beard, "Consensus seeking in multi-agent systems under dynamically changing interaction topologies," IEEE Trans. on Automatic Control, vol. 50, no. 5, pp. 655-661, 2005.

[36] C. G. J. Jacobi, Vorlesungen über Dynamik. Reimer, Berlin, 1866.

[37] R. Abraham and J. Marsden, Foundations of Mechanics, 2nd Ed. Addison-Wesley, 1978.

[38] F. Zhang, M. Goldgeier, and P. S. Krishnaprasad, "Control of small formations using shape coordinates," in Proc. of 2003 International Conf. of Robotics and Automation. Taipei, Taiwan: IEEE, 2003, pp. 2510-2515.

[39] A. Micaelli and C. Samson, "Trajectory tracking for unicycle-type and two-steering-wheels mobile robots," INRIA report 2097, 1993.

[40] C. Samson, "Control of chained systems: Application to path-following and time-varying point-stabilization of mobile robots," IEEE Trans. on Automatic Control, vol. 40, no. 1, pp. 64-77, 1995.

[41] K. Li and J. Baillieul, "Data-rate requirements for nonlinear feedback control," in 6th IFACS Symposium on Nonlinear Control Systems (NOLCOS), 2004.

[42] A. Balluchi, A. Bicchi, and P. Soueres, "Path-following with a bounded-curvature vehicle: a hybrid control approach," International Journal of Control, vol. 78, no. 15, pp. 1228-1247, 2005.

[43] R. Stengel, Optimal Control and Estimation. New York: Dover Publications, 1994.

[44] B. O. Anderson and J. B. Moore, Optimal Filtering. New Jersey: Prentice-Hall, 1979.

[45] D. Simon, Optimal State Estimation. New Jersey: Wiley, 2006.

[46] A. E. Bryson Jr. and L. J. Henrickson, "Estimation using sampled data containing sequentially correlated noise," AIAA Journal of Spacecraft and Robotics, vol. 5, no. 6, pp. 662-665, 1968.

[47] R. A. Horn and C. R. Johnson, Matrix Analysis. Cambridge University Press, New York, 1985.

[48] F. Zhang, E. Fiorelli, and N. E. Leonard, "Exploring scalar fields using multiple sensor platforms: Tracking level curves," in Proc. of 46th IEEE Conf. on Decision and Control, New Orleans, LA, 2007, pp. 3579-3584.

[49] R. S. Millman and G. D. Parker, Elements of Differential Geometry. Prentice-Hall, 1977.

[50] R. Littlejohn and M. Reinsch, "Internal or shape coordinates in the $n$-body problem," Physical Review A, vol. 52, no. 3, pp. 2035-2051, 1995.

[51] _ _ "Gauge fields in the separation of rotation and internal motions in the n-body problem," Reviews of Modern Physics, vol. 69, no. 1, pp. 213-275, 1997.

[52] E. Belbruno, Capture Dynamics and Chaotic Motions in Celestial Mechanics. Princeton, NJ: Princeton University Press, 2004.

[53] P. Ogren, M. Egerstedt, and X. Hu, “A control Lyapunov function approach to multiagent coordination,” IEEE Transactions on Robotics and Automation, vol. 18, no. 5, pp. 847-851, 2002.

[54] Y. Liu and K. M. Passino, "Stability analysis of m-dimensional asynchronous swarms with a fixed communication topology," IEEE Transactions on Automatic Control, vol. 48, no. 1, pp. 76-95, 2003.

[55] H. Khalil, Nonlinear Systems, 3rd Ed. New Jersey: Prentice Hall, 2001.

[56] Adaptive Sampling and Prediction (ASAP) Project. [Online]. Available: http://www.princeton.edu/ dcsl/asap/

[57] P. Lermusiaux, C. Evangelinos, R. Tian, P. Haley, J. McCarthy, N. Patrikalakis, A. Robinson, and H. Schmidt, "Adaptive coupled physical and biogeochemical ocean predictions: A conceptual basis," in Computational Science - ICCS 2004, Part 3, ser. Lecture Notes in Computer Science, 2004, vol. 3038, pp. 685-692. 